Three-dimensional spacetimes of maximal order

This article has been downloaded from IOPscience. Please scroll down to see the full text article.

2013 Class. Quantum Grav. 30095004

(http://iopscience.iop.org/0264-9381/30/9/095004)

View the table of contents for this issue, or go to the journal homepage for more

Download details:

IP Address: 157.193.117.112

The article was downloaded on 06/09/2013 at 16:49

Please note that terms and conditions apply. 


\title{
Three-dimensional spacetimes of maximal order
}

\author{
R Milson ${ }^{1}$ and L Wylleman ${ }^{2,3,4}$ \\ ${ }^{1}$ Department of Mathematics and Statistics, Dalhousie University, Halifax, Canada \\ 2 Department of Mathematical Analysis, Ghent University, B-9000 Ghent, Belgium \\ ${ }^{3}$ Mathematical Institute, Department of Mathematics, Utrecht University, 3584 CD Utrecht, \\ The Netherlands \\ ${ }^{4}$ Department of Mathematical and Natural Sciences, University of Stavanger, N-4036 Stavangar, \\ Norway \\ E-mail: robert.milson@dal.ca and lode.wylleman@ugent.be
}

Received 22 December 2012, in final form 2 January 2013

Published 11 April 2013

Online at stacks.iop.org/CQG/30/095004

\begin{abstract}
We show that the equivalence problem for three-dimensional Lorentzian manifolds requires at most the fifth covariant derivative of the curvature tensor. We prove that this bound is sharp by exhibiting a class of 3D Lorentzian manifolds which realize this bound. The analysis is based on a three-dimensional analogue of the Newman-Penrose formalism, and spinorial classification of the three-dimensional Ricci tensor.
\end{abstract}

PACS numbers: 02.40.Ky, 04.60. $-\mathrm{m}$

\section{Introduction and main result}

We report on recent progress concerning the invariant classification problem for threedimensional Lorentzian geometries. In a physical context, such geometries arise as exact solutions of three-dimensional theories of gravity, such as topologically massive gravity (TMG), new massive gravity (NMG) and extensions of those. We refer to [9] and the introduction of [1] for reviews of the relevant literature. In [9] it was stressed that, when surveying the literature of exact solutions, it is often difficult to disentangle genuinely new solutions from those that are already known but written in different coordinate systems.

To tackle this problem one needs a coordinate invariant local characterization of the geometry. A first step is to use the algebraic classification of the Ricci tensor, as was done in [9] to classify all TMG solutions known at that time. A complete answer to the problem (in any dimension in principle) is provided by the Cartan-Karlhede algorithm [8, 15]. The key quantities used here are so-called Cartan invariants, which are components of the Riemann tensor and a finite number of its covariant derivatives, relative to some maximally fixed vector frame associated to these tensors.

Regarding three-dimensional Lorentzian geometries, we will show in this paper that cases where one needs the theoretically maximal number of five derivatives for a complete 
classification do exist, but are limited to the metrics given in our main theorem 1 below. This implies that

any three-dimensional geometric theory of gravity whose field equations exclude the metrics of theorem 1 requires at most four covariant derivatives of the Riemann tensor for a complete local invariant classification of its exact solutions.

In the remainder of this introduction, we will outline the general mathematical context and background for the main theorem.

Let $(M, g)$ be a smooth, $n$-dimensional pseudo-Riemannian manifold, and let $(V, \eta)$ be a real inner-product space having the same dimension and signature as $(M, g)$. Henceforth, we use $\eta_{a b}$ to raise and lower frame indices, which we denote by $a, b, c=1, \ldots, n$. Let $O(\eta)$ be the group of automorphisms of $\eta$, and let $\mathfrak{o}(\eta)$ be the corresponding Lie algebra of anti self-dual transformations. An $\eta$-orthogonal coframe is an inner-product isomorphism

$$
\omega_{x}:\left(T_{x} M, g_{x}\right) \rightarrow(V, \eta), \quad x \in M .
$$

Let $\pi: O(\eta, M) \rightarrow M$ denote the principal $O(\eta)$-bundle of all such. An $\eta$-orthogonal moving coframe is a local section of this bundle, or equivalently, a collection of 1-forms $\omega^{a}$ such that

$$
g=\eta_{a b} \omega^{a} \omega^{b} .
$$

Set

$$
\mathcal{R}^{p}=\otimes^{4} V^{*} \oplus \cdots \oplus \otimes^{4+p} V^{*}
$$

and let $\hat{R}^{(p)}: O(\eta, M) \rightarrow \mathcal{R}^{p}$ be the canonical, $O(\eta)$-equivariant map defined by

$$
\hat{R}^{(p)}=\left(\hat{R}_{a b c d}, \hat{R}_{a b c d ; e}, \ldots, \hat{R}_{a b c d ; e_{1} \ldots e_{p}}\right),
$$

where the right hand side denotes the lift of the Riemann curvature tensor and its first $p$ covariant derivatives to $O(\eta, M)$.

The following definitions are adapted from [20, definitions 8.14 and 8.18]. Set $r_{-1}=0$, and let $r_{p}$ denote the rank of $\hat{R}^{(p)}, p=0,1,2, \ldots$ We say that $(M, g)$ is fully regular if $r_{p}$ is constant for all $p$. Henceforth we assume that full regularity holds and let $q=q_{M}$ be the smallest integer such that $r_{q-1}=r_{q}$. The integer $q-1$ is called the order of the metric $[20,26]$. It can be shown [20, theorem 12.11] that a fully regular metric of order $q-1$ is classified by $\hat{R}^{(q)}$, that is by $q$ th-order differential invariants.

The maximal order of a pseudo-Riemannian manifold, of fixed dimension and signature, is of particular interest. Cartan [8] established the upper bound

$$
q \leqslant n(n+1) / 2=\operatorname{dim} O(\eta, M) .
$$

Karlhede [15] improved Cartan's bound to

$$
q \leqslant n+s_{0}+1,
$$

where $s_{0}$ is the dimension of the automorphism group of the curvature tensor. The question of maximal order has received considerable attention in general relativity $(n=4$, Lorentzian signature) $[10,14,23]$. In that context, Karlhede's bound is $q \leqslant 7$; recently, this bound was shown to be sharp [18]. The four-dimensional metrics of maximal order describe a well-defined class of type $N$ spacetimes with aligned null-radiation in an anti-deSitter background [21]. By contrast, Karlhede's bound in the generic Petrov type I case (for which $s_{0}=0$ ) is $q \leqslant 5$, but at present we only have an example of a type I dust solution [29] with $q=3$.

In this paper, we investigate and classify three-dimensional Lorentzian manifolds of maximal order. Our approach is grounded in Karlhede's refinement of the Cartan equivalence method [22], which is based on the notion of curvature normalization [15, 26]. A non-zero 
three-dimensional curvature tensor has vanishing Weyl part and is thus represented by its Ricci tensor, which may be regarded as a self-adjoint operator on the three-dimensional tangent space. Generically, the Ricci operator has a finite automorphism group (whence $s_{0}=0$ ). However, if two eigenvalues coincide or if the trace-free part of the operator is nilpotent, then $s_{0}=1$ is possible. Therefore, in the three-dimensional Lorentzian setting, Karlhede's bound is $q \leqslant 5$ [25]. The question then becomes:

Does there exist a fourth order, three-dimensional Lorentzian metric, that is to say, a metric that is classified by fifth-order differential invariants?

In three-dimensional Lorentzian geometry, it is useful to make use of the real spinor representation of the Lorentz group. Such a spinor approach provides one with a natural null vector frame formalism. Moreover, the Petrov-Penrose classification of the curvature spinor (which, in three dimensions, is equivalent to the null alignment classification of the Ricci tensor) leads to a slight refinement of the usual Ricci-Segre classification. This is summarized in the appendices.

Karlhede's result, which we formulate as theorem 5 below, tells us that a metric which is classified by fifth order invariants, if one exists, is restricted to Petrov type D, type DZ (like type $\mathrm{D}$, but the doubly aligned null directions are complex) and type $\mathrm{N}$ geometries. Below, we rule out the type DZ and N possibilities, and demonstrate that the $q=5$ bound is realized for one very particular class of type $\mathrm{D}$ metrics.

Theorem 1. The order of a curvature-regular, three-dimensional Lorentzian manifold is bounded by

$$
q-1 \leqslant 4 .
$$

This bound is sharp; every fourth order metric is locally isometric to

$$
\begin{aligned}
& 2(2 T x \mathrm{~d} u+\mathrm{d} w)^{2}-2 \mathrm{~d} u(\mathrm{~d} x+a \mathrm{~d} u), \quad \text { where } \\
& a=\frac{1-\mathrm{e}^{4 T w}}{2 T}+\left(2 T^{2}-C\right)\left(x-\delta_{C}\right)^{2}+F(u) .
\end{aligned}
$$

Here $x, u, w$ are local coordinates. $C, T$ are real constants such that $C+2 T^{2} \neq 0$, and $F(u)$ is an arbitrary real function such that

$$
\begin{cases}(1+2 T F(u)) F^{\prime \prime}(u) \neq 3 T\left(F^{\prime}(u)\right)^{2} & \text { if } C \neq 0 \\ F^{\prime}(u) \neq 0 & \text { if } C=0\end{cases}
$$

Note 1 . In the singular subcase of $T=0$, the expression $\left(1-\mathrm{e}^{4 T w}\right) /(2 T)$ should be interpreted in the limit sense as being equal to $-2 w$.

Note 2. The expression $\delta_{C}$ denotes 1 if $C=0$ and 0 if $C \neq 0$.

Note 3. Curvature regularity is a strengthening of the full-regularity assumption that we impose in order to exclude 'type-changing' metrics (see definition 2 below).

The structure of this paper is as follows. In section 2 we revise the relevant definitions and theorems regarding curvature normalization, leading to Karlhede's bound within his approach to the equivalence problem. The concepts of curvature homogeneity and pseudo-stabilization turn out to be the crucial ideas in the search for metrics of maximal order. In particular, the maximal order metrics shown in (4) enjoy the $\mathrm{CH}_{1}$ (curvature homogeneous of order 1) property. The relevant definitions are given in section 3 . We isolate the structure equations for the maximal order metrics in section 4 . We then prove the main theorem 1 by integrating these equations in section 5. Relevant background material is put in four appendices: a threedimensional analogue of the Newman-Penrose (NP) formalism, the transformation rules of connection and curvature variables under basic Lorentz transformations, the Petrov-Penrose classification of the three-dimensional Ricci tensor, and the structure equations obeyed by a $\mathrm{CH}_{1}$ metric. 


\section{Curvature normalization and Karlhede's bound}

A general approach towards finding metrics of maximal order was described in [10] and [19]. The approach is based on two key ideas: (i) curvature normalization, also known as the Karlhede algorithm [15], and (ii) curvature homogeneity [24]. Normalization of the curvature tensor and its covariant derivatives, also known as the Karlhede algorithm, splits the rank of the classifying map $\hat{R}^{(p)}$ into horizontal and vertical subranks and thereby simplifies the equivalence problem. As was already mentioned, the rank $r_{p}$ is the maximal number of functionally independent component functions $\left(\hat{R}_{a b c d}, \ldots, \hat{R}_{a b c d ; e_{1} \ldots e_{p}}\right)$, where the latter are functions of both position and frame variables. In order to speak of horizontal rank, we need to assume that the above tensors can be normalized. The horizontal rank (see definition 3 below) can then be defined as the maximal number of functionally independent component functions of normalized curvature and its covariant derivatives.

Definition 2. We say that a submanifold $S \subset \mathcal{R}^{p}$ is a pth order normalizing cross-section for $(M, g)$ provided:

(N1) there exists a subgroup $G_{p} \subset O(\eta)$ that fixes $S$ pointwise;

(N2) the normalization is maximal in the sense that $X(S) \cap S \neq \emptyset, X \in O(\eta)$ implies $X \in G_{p}$

(N3) $(M, g)$ admits a cover by $\eta$-orthonormal moving coframes such that

$$
\operatorname{img} R^{(p)} \subset S, \quad \text { where } R^{(p)}=\left(R_{a b c d}, \ldots, R_{a b c d ; e_{1} \ldots e_{p}}\right)
$$

denotes the curvature components relative to the coframe in question.

If there exists a normalizing cross-section $S \subset \mathcal{R}^{p}$ for every $p=0,1,2, \ldots$ we say that $(M, g)$ is curvature regular.

Suppose that curvature regularity holds. Normalizing $R^{(p)}$ reduces the structure group of the equivalence problem from $O(\eta)$ to $G_{p}$. Because of $\mathrm{N} 2$, the maximally normalized components $\left(R_{a b c d}, \ldots, R_{a b c d ; e_{1} \ldots e_{p}}\right)$ are locally defined functions on the base $M$. These differential invariants, commonly referred to as $p$ th order Cartan invariants, suffice to invariantly classify $(M, g)$ and to solve the metric equivalence problem [26, chapter 9].

Definition 3. Suppose that $(M, g)$ is curvature regular. We define

$$
\begin{aligned}
s_{p} & :=\operatorname{dim} G_{p}, \\
t_{p} & :=\operatorname{rank} R^{(p)}
\end{aligned}
$$

relative to some choice of normalizing cross-section. We refer to $s_{p}$ as the pth order degree of frame freedom, and to $t_{p}$ as the pth order horizontal rank.

Proposition 4. If $(M, g)$ is curvature regular, then $s_{p}, t_{p}$ do not vary with $x \in M$ and are independent of the choice of normalizing cross-section. Furthermore,

$$
s_{p} \leqslant s_{p-1}, \quad t_{p} \geqslant t_{p-1}
$$

and

$$
r_{p}=t_{p}+n(n-1) / 2-s_{p}, \quad p=0,1,2, \ldots
$$


Theorem 5 (Karlhede, theorem 4.1 of [15], see also section 9.2 of [26]). Let $(M, g)$ be a fully regular, curvature regular $n$-dimensional pseudo-Riemannian manifold with isometry group $K$. Let $r_{p}, t_{p}, s_{p}$ be as defined above, and let $q$ be the smallest integer such that $r_{q-1}=r_{q}$. Then, $q$ is also the smallest integer such that $s_{q-1}=s_{q}$ and $t_{q-1}=t_{q}$. Furthermore, we have that $G_{q-1} \subset O(\eta)$ is isomorphic to the isotropy subgroups $K_{x} \subset O\left(T_{x} M\right), x \in M$; that

$$
\operatorname{dim} K=n-t_{q}+s_{q},
$$

and that $n-t_{q}$ is equal to the dimension of the $K$-orbits.

In particular, (10) implies that

$$
r_{0} \geqslant n(n-1) / 2-s_{0} .
$$

By the regularity assumption,

$$
r_{0}+p \leqslant r_{p} \leqslant n(n+1) / 2, \quad 0 \leqslant p \leqslant q-1 .
$$

Applying the above inequality with $p=q-1$ and using (12) gives the Karlhede bound (3) as an immediate corollary.

\section{Curvature homogeneity and pseudo-stabilization}

Suppose that $(M, g)$ is fully regular and curvature regular. The curvature homogeneity condition admits several equivalent definitions $[4,12]$, but with the above assumptions, the following definition is the most convenient.

Definition 6. A manifold $(M, g)$ is curvature-homogeneous of order $k$, or $\mathrm{CH}_{k}$ for short, if it is curvature regular and if the horizontal rank $t_{k}=0$. If $t_{k}=0$ and $t_{k+1}>0$, we say that $(M, g)$ is properly $\mathrm{CH}_{k}$.

To put it another way, a properly curvature homogeneous manifold of order $k$ has constant Cartan invariants of order $\leqslant k$, with a non-constant invariant appearing at order $k+1$. The main application of the curvature homogeneous concept was the following theorem [24].

Theorem 7 (Singer). A manifold $(M, g)$ is locally homogeneous if and only if it is $\mathrm{CH}_{k}$ for all $k=0,1,2, \ldots$.

In other words, a locally homogeneous space is characterized by the property of having constant Cartan invariants. As such, Singer's theorem is an immediate corollary of theorem 5.

In this paper we are interested in curvature homogeneity for a different, but related reason. As was shown in [18], curvature homogeneity is also a key concept in the search for maximal order metrics. The relevant observation is that for a $\mathrm{CH}_{k}$ geometry the rank $r_{k}$ is small because $t_{k}=0$, and this is exactly what is needed for maximal order. Let us explain further in the context of three-dimensional Lorentzian metrics.

Definition 8. We say that a curvature regular geometry has kth order pseudo-stabilization provided $s_{k}=s_{k-1}>s_{q}$.

Our notion of pseudo-stabilization is different but conceptually related to the notion employed in [20, theorem 5.37]. Notice that a $k$ th order pseudo-stable geometry has $t_{k}>t_{k-1}$ by theorem 5 .

Proposition 9. A fourth order, three-dimensional, Lorentz geometry, if one exists, is either properly $\mathrm{CH}_{1}$ or is properly $\mathrm{CH}_{0}$ with first order pseudo-stabilization. 
Proof. Table $\mathrm{C} 1$ reveals $s_{0} \leqslant 1$ for a non-homogeneous geometry (see proposition 10 below). Hence, $r_{0} \geqslant 2$, and hence a fourth order geometry requires the following rank sequence:

$$
\left(r_{p}\right)=(2,3,4,5,6,6) .
$$

This can be achieved in essentially two ways: either by

$$
\left(t_{p}\right)=(0,0,1,2,3,3), \quad\left(s_{p}\right)=(1,0,0,0,0,0),
$$

which describes a properly $\mathrm{CH}_{1}$ geometry, or by three possible sequences with $s_{q}=0$ and starting with

$$
\left(t_{p}\right)=(0,1, \ldots), \quad\left(s_{p}\right)=(1,1, \ldots),
$$

which describes a properly $\mathrm{CH}_{0}$ geometry with first order pseudo-stabilization.

In the following section, we rule out the pseudo-stabilization and type DZ, N scenarios and show that a fourth order requires a type $\mathrm{D}$, properly $\mathrm{CH}_{1}$ geometry. We then explicitly write down the necessary structure equations and integrate them. The end result is theorem 1.

\section{The equivalence problem}

In this section we derive the necessary and sufficient conditions for a fourth order metric. Table C1 of the appendix shows that $s_{0}>0$ for curvature types O, N, DZ, and D. Type O can be ruled out by Schur's theorem. A proof can be found in [28, corollaries 2.2.5 and 2.2.7].

Proposition 10. If the curvature is type $O$ at all points $x \in M$, then $M$ is a locally homogeneous space, i.e. $t_{p}=0$ for all $p$.

We are left with the following possibilities.

Proposition 11. A fourth order metric, if one exists, requires curvature of type $N, D Z$, or D.

According to proposition 9, each of the above three cases further splits into two subcases, according to whether the geometry is properly $\mathrm{CH}_{1}$ or properly $\mathrm{CH}_{0}$ with first order pseudostabilization. We consider the above possibilities in turn. Five of the possibilities can be ruled out, and this leaves a unique configuration for a fourth order metric.

Since in a $\mathrm{CH}_{0}$ geometry the zeroth order components $R_{a b c d}$ are constant, the first order components $R_{a b c d ; e}$ are quadratic expressions of certain spin coefficients. Therefore, in the analysis that follows it is more convenient to specify the Cartan invariants in terms of spin coefficients and their frame derivatives. This methodology for constructing invariants is related to the notion of essential torsion in the Cartan equivalence method. The relevant details and definitions are given in appendix D.

\subsection{Type $N$ configurations}

Taking the curvature canonical form of table $\mathrm{C} 1$ for this case, and assuming the $\mathrm{CH}_{0}$ property, we have

$$
\Psi_{0}=\Psi_{1}=\Psi_{2}=\Psi_{3}=0, \quad \Psi_{4}= \pm 1, \quad R=\tilde{R},
$$

where $\tilde{R}$ is a real constant. The group $G_{0}$ preserving (15) is generated by null rotations (B.17) about $\ell$ and the reflections (B.45), (B.48). The type $\mathrm{N}$ first order torsion matrix (see appendix D for the derivation) is

$$
\left(\Gamma_{a}^{\rho}\right)=\left(\begin{array}{lll}
\kappa & \sigma & \tau \\
\epsilon & \alpha & \gamma
\end{array}\right) .
$$

6 
Substituting (15) into the Bianchi equations (A.45)-(A.47) yields the relations

$$
\kappa=0, \quad \sigma=2 \epsilon .
$$

We now consider the $\mathrm{CH}_{1}$ and pseudo-stable cases in turn.

Proposition 12. A type $N$, properly $\mathrm{CH}_{1}$ geometry has order bounded by $q-1 \leqslant 3$.

Proof. By assumption, after the first-order torsion is normalized, $\epsilon, \tau, \alpha, \gamma$ are constants. Hence, by (A.36)-(A.40)

$$
\begin{aligned}
& \sigma=\epsilon=0, \quad R=-12 \tau^{2}, \\
& (\tau+\pi)(2 \alpha+\tau)=0 .
\end{aligned}
$$

By (B.19)-(B.24), $\tau$ and $\alpha$ are invariant under any null rotation about $\ell$, while $\gamma$ transforms like

$$
\gamma^{\prime}=\gamma+x(2 \alpha+\tau)
$$

Since $t_{0}=t_{1}=0$ and $s_{0}=1$ by assumption, $s_{1}=1$ would lead to $q-1=0$. Thus we assume $s_{1}=0$ henceforth. By (20) this entails $2 \alpha+\tau \neq 0$, and hence

$$
\pi=-\tau
$$

by (19). We impose the normalizations

$$
\gamma=0, \quad 2 \alpha+\tau>0,
$$

which leaves $G_{1}$ as the discrete group generated by (B.45). Then equation (A.42) implies that

$$
\lambda=0 \text {. }
$$

From (21), (23) and $s_{1}=0$ it follows that the second order invariants are generated by $v$. If $v$ is constant then $t_{2}=0$ and $q-1=1$. Thus we assume henceforth that $v$ is non-constant, i.e. $t_{2}=1$. The remaining structure equations (A.43) and (A.44) reduce to

$$
D v=0, \quad \delta v=2 v(\tilde{\tau}-2 \tilde{\alpha}) \pm 1 / 2,
$$

where $\tilde{\tau}, \tilde{\alpha}$ are constants such that $2 \tilde{\alpha}+\tilde{\tau}>0$. Suppose then that $\Delta v$ is functionally independent from $v$, and hence that $t_{3}=2$ (else $t_{3}=1$ and $q-1=2$ ). By the (N2) curvature regularity assumption we have $\Delta v \neq 0$ at each point and we fully fix the frame by normalizing

$$
\Delta v>0 \text {. }
$$

Now the third order invariants are generated by $v, \Delta v$. Applying (A.20) and (A.21) to $v$ gives

$$
D \Delta v=0, \quad \delta \Delta v=3(\tilde{\tau}-2 \tilde{\alpha}) \Delta v .
$$

Hence, the fourth order invariants are generated by $v, \Delta v$ and $\Delta^{2} v$. Applying (A.20) to $\Delta v$ gives

$$
D \Delta^{2} v=0
$$

and hence

$$
\mathrm{d} v \wedge \mathrm{d} \Delta v \wedge \mathrm{d} \Delta^{2} v=0
$$

Therefore $t_{4}=2$, which implies that the order is $q-1=3$.

Proposition 13. The order of a type $N, \mathrm{CH}_{0}$, pseudo-stable geometry is bounded by $q-1 \leqslant 3$. 
Proof. Referring to (16), the assumption $s_{1}=1$ implies that the remaining torsion scalars $\epsilon, \tau, \alpha$ and $\gamma$ are invariant under arbitrary null rotations about $\ell$ and thus generate the first order Cartan invariants. By (B.21)-(B.24) this implies

$$
\epsilon=\sigma=0, \quad \alpha=-\tau / 2 .
$$

Hence the first order invariants $R_{a b c d ; e}$ are generated by $\tau, \gamma$. By the pseudo-stabilization assumption $R_{a b c d ; e}$ is $G_{0}$-invariant, and hence, using the notation of appendix D,

$$
R_{a b c d ; e f}=(\nabla R)_{a b c d e, f}+\Gamma_{f}^{\rho}\left(\boldsymbol{A}_{\rho} \cdot \nabla R\right)_{a b c d e f} .
$$

It follows that the second order components are linear combinations of $D \gamma, D \tau$, $\delta \tau, \Delta \tau, \delta \gamma, \Delta \gamma$ and quadratic polynomials of $\tau, \gamma$. Since the latter are null-rotation invariant, the second order Cartan invariants are obtained by normalizing the former.

From here equations (A.36)-(A.40) reduce to

$$
D \tau=0, \quad \delta \tau=-D \gamma=\tilde{R} / 12+\tau^{2} .
$$

If $t_{1}>1$ then $q-1 \leqslant 3$ automatically, so we may assume $t_{1}=1$. This implies $\mathrm{d} \tau \wedge \mathrm{d} \gamma=0$, and in particular $D \gamma \delta \tau=0$. Hence,

$$
D \gamma=\tilde{R}+12 \tau^{2}=0,
$$

which implies that $\tau=\tilde{\tau}$ is a constant. This leaves $\gamma$ as the only generator of the first order invariants. The transformation law (B.17) gives

$$
\Delta^{\prime} \gamma=\Delta \gamma-4 x \gamma \tilde{\tau}
$$

At this point, we must consider two cases.

Case (a). Suppose that $\tilde{\tau}=0$ Hence, $\Delta \gamma$ is null-rotation invariant, and hence is a Cartan invariant. By (A.42),

$$
\delta \gamma=0
$$

Since $\gamma, \Delta \gamma$ generate the second order invariants, we have $s_{2}=1$. Applying (A.20) and (A.21) to $\gamma$ gives

$$
D \Delta \gamma=\delta \Delta \gamma=0
$$

Hence, $\mathrm{d} \gamma \wedge \mathrm{d} \Delta \gamma=0$. This implies $t_{2}=1$ and thus $q=2$. Hence, the corresponding geometries are not pseudo-stable.

Case (b). Suppose that $\tau \neq 0$. In view of (28), (B.46) and (B.49) we may fully fix the frame $\left(s_{2}=0\right)$ by imposing the normalizations

$$
\gamma>0, \quad \tau>0, \quad \Delta \gamma=0 .
$$

By (A.42),

$$
\delta \gamma=2 \tilde{\tau} \gamma
$$

It follows that $t_{2}=1$, and that the first and second order invariants are generated by $\gamma$. Again, using the notation of appendix D,

$$
R_{a b c d ; e_{1} e_{2} f}=\left(\nabla^{2} R\right)_{a b c d e_{1} e_{2}, f}+\Gamma_{f}^{\alpha}\left(\boldsymbol{A}_{\alpha} \cdot \nabla^{2} R\right)_{a b c d e_{1} e_{2}} .
$$

By (27), (30), (31), the components $\left(\nabla^{2} R\right)_{a b c d e_{1} e_{2}, f}$ are generated by $\gamma$. Since the automorphism group of $\nabla^{2} R$ is trivial, equations (32) can be solved for $\Gamma^{\alpha}{ }_{f}$. It follows that $\lambda, v, \pi$, together with $\gamma$, generate invariants of order 3 or less. The commutator relations (A.20) and (A.21) applied to $\gamma$ give

$$
\gamma \tilde{\tau}(\pi+\tilde{\tau})=0, \quad \gamma \tilde{\tau} \lambda=0 .
$$


Hence,

$$
\pi=-\tilde{\tau}, \quad \lambda=0 .
$$

The remaining structure equations (A.43) and (A.44) reduce to

$$
D v=0, \quad \delta v=4 v \tilde{\tau} \pm 1 / 2 .
$$

Observe that $d \gamma \wedge d \nu=0$ if and only if $\Delta v=0$; the corresponding rank sequence is

$$
\left(s_{p}\right)=(1,1,0,0), \quad\left(t_{p}\right)=(0,1,1,1),
$$

and the order is $q-1=2$. Else we have $t_{3}=2$, the fourth order invariants being generated by $\gamma, v$ and $\Delta v$. Applying (A.20) to $v$ gives $D \Delta v=0$ and thus

$$
\mathrm{d} \gamma \wedge \mathrm{d} v \wedge \mathrm{d} \Delta v=0
$$

Hence, the rank sequence is

$$
\left(s_{p}\right)=(1,1,0,0,0), \quad\left(t_{p}\right)=(0,1,1,2,2),
$$

and the order is equal to $q-1=3$.

\subsection{Type DZ configurations}

For this case, combining the curvature canonical form of table $\mathrm{C} 1$ and the $\mathrm{CH}_{0}$ property $t_{0}=0$ gives

$$
\Psi_{1}=\Psi_{3}=0, \quad \Psi_{0}=\Psi_{4}=3 \Psi_{2}=3 \tilde{\Psi}_{2} \neq 0, \quad R=\tilde{R}
$$

where $\tilde{\Psi}_{2}$ and $\tilde{R} \in \mathbb{R}$ are real constants. The group $G_{0}$ preserving (35) is generated by spins (B.33) and the reflections (B.45), (B.48), (B.51). The first order torsion is

$$
\left(\Gamma_{a}^{\rho}\right)=\left(\begin{array}{ccc}
\epsilon & \alpha & \gamma \\
\kappa-\pi & \sigma-\lambda & \tau-v
\end{array}\right) .
$$

Substituting (35) into the Bianchi equations (A.45)-(A.47) yields

$$
\sigma-\lambda=-2 \gamma=2 \epsilon, \quad \kappa-\pi=-(\tau-\nu) \text {. }
$$

Proposition 14. There does not exist a type DZ, $\mathrm{CH}_{0}$ geometry with pseudo-stabilization.

Proof. The assumption implies that $t_{0}=0, t_{1}>0$ and that the first order torsion is spininvariant. By (B.38), this entails

$$
\sigma-\lambda=\gamma=\epsilon=0, \quad \kappa-\pi=-(\tau-v)=2 \alpha .
$$

Hence, $\alpha$ generates the first order invariants. However, (A.40) entails

$0=2(\tau+\kappa-2 \alpha) \alpha+(\kappa-2 \alpha) \tau-\kappa(2 \alpha+\tau)+\Psi_{2}-R / 12=-4 \alpha^{2}+\tilde{\Psi}_{2}-\tilde{R} / 12$

This implies that $\alpha$ is a constant, which contradicts the $t_{1}>0$ assumption.

Proposition 15. A type DZ, properly $\mathrm{CH}_{1}$ geometry does not exist.

Proof. In addition to (35) we assume that $t_{1}=0, t_{2}>0$ and that $s_{1}=0$. The $t_{1}=0$ assumption means that post-normalization, the torsion components (36) are constant, say

$$
-(\kappa-\pi)=\tau-v=C_{1}, \quad \sigma-\lambda=-2 \gamma=2 \epsilon=C_{2}, \quad \alpha=\tilde{\alpha} .
$$

Transformation law (B.38) now reads

$$
\left(C_{1}+2 \alpha+2 \mathrm{i} C_{2}\right)^{\prime}=\mathrm{e}^{ \pm 2 \mathrm{it}} \text { lhs. }
$$


Since $s_{1}=0$ this cannot be zero and we may therefore impose the normalization

$$
C_{2}=0, \quad C_{1}+2 \tilde{\alpha}>0 .
$$

Applying (40), (37) to equation (A.38) gives

$$
\sigma\left(C_{1}+2 \tilde{\alpha}\right)=0 .
$$

Then, (A.36)-(A.44) entail

$$
\sigma=\lambda=0, \quad \pi=\tau=-v=-\kappa=C_{1} / 2 .
$$

Hence, all second order Cartan invariants are constant, a contradiction.

\subsection{Type D configurations}

Proposition 16. There does not exist a type $D$, properly $\mathrm{CH}_{0}$ geometry with pseudostabilization.

Proof. The curvature normalization from table $\mathrm{C} 1$ and the $\mathrm{CH}_{0}$ assumption give

$$
\Psi_{0}=\Psi_{1}=\Psi_{3}=\Psi_{4}=0, \quad \Psi_{2}=\tilde{\Psi}_{2}, R=\tilde{R},
$$

where $\tilde{\Psi}_{2} \neq 0$ and $\tilde{R}$ are constants, and we also assume

$$
s_{0}=s_{1}=1, \quad t_{0}=0, t_{1}>0 .
$$

The first order torsion is

$$
\left(\Gamma_{a}^{\rho}\right)=\left(\begin{array}{lll}
\kappa & \sigma & \tau \\
\pi & \lambda & \nu
\end{array}\right)
$$

where, by the Bianchi relations,

$$
\sigma=\lambda=0, \quad \pi=\tau .
$$

By the boost transformation laws (B.3)-(B.8), in order to have $s_{1}=1$ we require

$$
\kappa=v=0 .
$$

Adding (A.39) to (A.41) yields

$$
0=2 \tau^{2}+\tilde{\Psi}_{2}-\tilde{R} / 6,
$$

which implies that $\tau$ is constant. Hence $t_{1}=0$, contradicting our assumption.

Proposition 17. Up to $O(\eta)$ conjugation, the unique type $D$, properly $\mathrm{CH}_{1}$ configuration is

$$
\begin{aligned}
& \Psi_{0}=\Psi_{1}=\Psi_{3}=\Psi_{4}=0, \quad \Psi_{2}=-\frac{2}{3}\left(C+2 T^{2}\right), \quad R=4\left(C-T^{2}\right), \\
& \sigma=\alpha=\gamma=\lambda=v=0, \quad \pi=\tau=T, \quad \kappa=1, \\
& \delta \epsilon=-T \epsilon, \quad \Delta \epsilon=C, \quad \epsilon>0,
\end{aligned}
$$

where $T$ and $C$ are constants such that $C+2 T^{2} \neq 0$.

Proof. Suppose that the curvature is type D, and that

$$
\left(t_{p}\right)=\left(0,0, t_{2}, \ldots\right), \quad t_{2}>0 .
$$

As above we have (42) and (43). The curvature automorphism group $G_{0}$ is generated by the one-dimensional group of boost transformations (B.1) and the discrete transformations (B.45), (B.48), (B.51). The corresponding transformation laws are shown in appendix B. Since $t_{0}=t_{1}=0$ this means that post-normalization, $R, \Psi_{2}, \kappa, \sigma, \tau, \pi, \lambda, \nu$ are all constant, 
where we put $\pi=\tau=T$. As above, the Bianchi identities give (44). Equations (A.36), (A.37), (A.43) and (A.44) reduce to

$$
\alpha \kappa=\gamma \kappa=\epsilon \nu=\alpha \nu=0 .
$$

If $\kappa=v \equiv 0$, identically, then by (B.3)-(B.8) the first order torsion is boost-invariant, which violates the assumption $s_{1}=0$. Suppose then that $\kappa \neq 0$. By the (N2) maximality assumption in definition 2, $\kappa$ cannot change sign. Using (B.7) and (B.49), we impose the normalization

$$
\kappa=1 \text {. }
$$

The second order torsion is $\alpha, \gamma, \epsilon$. If $v \neq 0$ then, by (48) the second order torsion vanishes, which violates the assumption $t_{2}>0$. Therefore,

$$
v=\alpha=\gamma \equiv 0
$$

identically. Hence, $t_{2}=1$. The case where $\kappa \equiv 0, v \neq 0$ does not need to be analysed, because it can be reduced to the present case by the Lorentz transformation (B.51), (B.52). Again by the (N2) assumption of maximal normalization, $\epsilon$ must have definite sign. Using (B.45), (B.46) to impose the normalization $\epsilon>0$ fully fixes the frame. Taking the second part of (45) as a definition for the constant $C$, equation (A.39) gives

$$
R=-6 \Psi_{2}-12 \tau^{2}=4\left(C-T^{2}\right) .
$$

The rest of (A.36)-(A.44) are either satisfied identically, or reduce to (47).

Above, we have derived a unique set of necessary conditions for a type $\mathrm{D}$ properly $\mathrm{CH}_{1}$ geometry. In other words, if such a metric exists, then around every point there exists a unique null-orthogonal moving frame such that (45)-(47) hold. Such geometries feature first order invariants $C, T$, which must be constants, and a unique, up to functional dependence, nonconstant second order invariant $\epsilon$. This is the necessity question. Next, we consider sufficiency.

The configuration equations (45)-(47) constitute a system of partial differential equations for type $\mathrm{D}$, properly $\mathrm{CH}_{1}$ metrics. We reformulate this system as the structure equations of a generalized Cartan realization problem [7, appendix] [11, section 3] using Bryant's recent treatment [6] of the realization problem. To wit, (45)-(47) is equivalent to

$$
\begin{aligned}
& \mathrm{d} \omega^{0}=-T \omega^{0} \wedge \omega^{1}, \\
& \mathrm{~d} \omega^{1}=-4 T \omega^{0} \wedge \omega^{2}, \\
& \mathrm{~d} \omega^{2}=\omega^{0} \wedge \omega^{1}+2 \epsilon \omega^{0} \wedge \omega^{2}-T \omega^{1} \wedge \omega^{2}, \\
& \mathrm{~d} \epsilon=P \omega^{0}-T \epsilon \omega^{1}+C \omega^{2}, \quad \text { where } P=D \epsilon .
\end{aligned}
$$

Proposition 18. Up to diffeomorphism, the general solution of (51)-(54) depends on one function of one variable.

Proof. Writing

$$
\mathrm{d} P=P_{1} \omega^{0}+(C-2 T P) \omega^{1}+2\left(C+2 T^{2}\right) \epsilon \omega^{2}, \quad \text { where } P_{1}=D^{2} \epsilon,
$$

a straightforward calculation shows that the differential ideal generated by (51)-(54) is closed; i.e., $\mathrm{d}^{2}=0$. The symbol tableau and its prolongation are

$$
A=\operatorname{span}\left(\begin{array}{lll}
1 & 0 & 0
\end{array}\right), \quad A^{(1)}=\operatorname{span}\left(\begin{array}{lll}
1 & 0 & 0 \\
0 & 0 & 0 \\
0 & 0 & 0
\end{array}\right) .
$$

Hence, the reduced characters are $c_{1}=1, c_{2}=0, c_{3}=0$, with

$$
c_{1}+2 c_{2}+3 c_{3}=1=\operatorname{dim} A^{(1)} .
$$

The tableau is involutive of rank 1 . The desired conclusion now follows by [6]. 
Proposition 19. Generically, the metric described by the preceding proposition is classified by fifth order invariants.

Proof. For generic solutions of (51)-(54), $\epsilon, P=D \epsilon, P_{1}=D^{2} \epsilon$ are functionally independent. We already observed that $\epsilon$ is a second order invariant. Hence $P, P_{1}$ are a third and a fourth order invariant, respectively. Generically, these will be functionally independent, and therefore, the rank sequence is as shown in (13).

\section{Three-dimensional metrics of maximal order}

In this section, we prove theorem 1 . Throughout, we assume full rank regularity and curvature regularity. By propositions 12-17, all fourth order metrics are necessarily type D and properly $\mathrm{CH}_{1}$. By propositions 18 and 19 such a geometry satisfies (51)-(54) and

$$
\mathrm{d} \epsilon \wedge \mathrm{d} P \wedge \mathrm{d} P_{1} \neq 0, \quad C+2 T^{2} \neq 0
$$

where

$$
P:=D \epsilon, \quad P_{1}:=D P=D^{2} \epsilon .
$$

We complete the proof of the main theorem 1 by integrating (51)-(54) subject to the constraints (56).

First assume $T \neq 0$. To integrate (51) we introduce an integrating factor:

$$
\mathrm{d}\left(\mathrm{e}^{-2 T w} \omega^{0}\right)=0 \text {. }
$$

Hence,

$$
\begin{aligned}
& \omega^{0}=\mathrm{e}^{2 T w} \mathrm{~d} u, \\
& \omega^{1}=2 \mathrm{~d} w+4 T x \mathrm{~d} u,
\end{aligned}
$$

for some functions $u, x, w$. Next, (52) gives

$$
\mathrm{d} \omega^{1}-4 T \omega^{2} \wedge \omega^{0}=4 T\left(\mathrm{e}^{-2 T w} \mathrm{~d} x-\omega^{2}\right) \wedge \omega^{0}=0,
$$

with general solution

$$
\omega^{2}=\mathrm{e}^{-2 T w}(\mathrm{~d} x+a \mathrm{~d} u) .
$$

Since $\omega^{0}, \omega^{1}, \omega^{2}$ are linearly independent, $u, w, x$ form a system of coordinates, and $a$ is some, as yet undetermined, function of $u, w, x$. Solving (58)-(60) gives

$$
\begin{aligned}
\mathrm{d} u & =\mathrm{e}^{-2 T w} \omega^{0}, \\
\mathrm{~d} w & =\frac{1}{2} \omega^{1}-2 T x \mathrm{e}^{-2 T w} \omega^{0}, \\
\mathrm{~d} x & =\mathrm{e}^{2 T w} \omega^{2}-a \mathrm{e}^{-2 T w} \omega^{0} .
\end{aligned}
$$

By (54), we have

$$
\mathrm{d}\left(\mathrm{e}^{2 T w} \epsilon-C x\right) \wedge \omega^{0}=0 .
$$

Hence,

$$
\epsilon=\mathrm{e}^{-2 T w}(C x+f(u)) .
$$

for some univariate function $f(u)$. Taking the exterior derivative of (60) and using (53) gives

$$
\left\{\mathrm{d} a+2 \mathrm{e}^{4 T w} \mathrm{~d} w+2\left(\left(C-2 T^{2}\right) x+f(u)\right) \mathrm{d} x\right\} \wedge \mathrm{d} u=0 .
$$

Making the substitution

$$
a=a_{1}+\frac{1-\mathrm{e}^{4 T w}}{2 T}+x^{2}\left(2 T^{2}-C\right)-2 x f(u)
$$


gives

$$
\mathrm{d} a_{1} \wedge \mathrm{d} u=0 .
$$

Therefore, for $T \neq 0$ the general solution of (51)-(54) is given by (58)-(60) and

$$
a=\frac{1-\mathrm{e}^{4 T w}}{2 T}+x^{2}\left(2 T^{2}-C\right)-2 x f(u)+f_{1}(u),
$$

where $f(u), f_{1}(u)$ are freely chosen functions. This solution form is invariant with respect to the following transformations:

$$
\begin{aligned}
& u=\phi(U), \quad w=W-\frac{\log \phi^{\prime}}{2 T}, \quad x=\frac{X}{\phi^{\prime}}+\frac{\phi^{\prime \prime}}{4 T^{2}\left(\phi^{\prime}\right)^{2}}, \\
& f(u)=\frac{F(U)}{\phi^{\prime}}-\frac{C \phi^{\prime \prime}}{4 T^{2}\left(\phi^{\prime}\right)^{2}}, \\
& f_{1}(u)=\frac{F_{1}(U)}{\left(\phi^{\prime}\right)^{2}}+\frac{2 F(U) \phi^{\prime \prime}-\phi^{\prime \prime \prime}}{4 T^{2}\left(\phi^{\prime}\right)^{3}}+\frac{\left(6 T^{2}-C\right)\left(\phi^{\prime \prime}\right)^{2}}{16 T^{4}\left(\phi^{\prime}\right)^{4}}+\frac{1-\left(\phi^{\prime}\right)^{2}}{2 T\left(\phi^{\prime}\right)^{2}},
\end{aligned}
$$

where $\phi(U)$ is an arbitrary strictly increasing function $\left(\phi^{\prime}(U)>0\right.$ everywhere).

If $T=0$ then one verifies that (51)-(54) is still equivalent to (58)-(63). Moreover, if $\left(1-\mathrm{e}^{4 T w}\right) /(2 T)$ is interpreted in the limit sense as being equal to $-2 w$, (66) remains valid. The form-preserving transformations are now

$$
\begin{aligned}
& u=U+U_{0}, \quad w=W+W_{0}, \quad x=X+\phi(U), \\
& f(u)=F(U)-C \phi, \quad f_{1}(u)=F_{1}(U)+2 F(U) \phi-C \phi^{2}-\phi^{\prime}+2 W_{0},
\end{aligned}
$$

where $U_{0}, W_{0}$ are constants and $\phi(U)$ is an arbitrary function.

It follows by (68) and (71) that if $C \neq 0$, then one can normalize the above solution form by transforming $f(u) \rightarrow 0$ identically. If $C=0$ then $T \neq 0$ by assumption, and hence by (62) and (68) one can normalize the solution form by transforming $f(u) \rightarrow 2 T^{2}$. Evaluating $\frac{1}{2}\left(\omega^{1}\right)^{2}-2 \omega^{0} \omega^{2}$ gives the metric in (4). Finally, a straightforward calculation relative to this metric form shows that the maximal order condition (56) is equivalent to (6).

The above maximal order metrics are invariantly classified by the invariant scalars $C, T$ and by the following Cartan invariants of orders 2, 3, 4, 5, respectively:

$$
\epsilon, P=D \epsilon, P_{1}=D^{2} \epsilon, P_{2}=D^{3} \epsilon .
$$

If $C \neq 0$, it is convenient to introduce the invariants

$J:=\delta \epsilon \Delta P-\delta P \Delta \epsilon=2 C T P-2 T\left(C+2 T^{2}\right) \epsilon^{2}-C^{2}$,

$J_{1}:=D \epsilon \Delta P-D P \Delta \epsilon=-C P_{1}+2\left(C+2 T^{2}\right) \epsilon P$,

$J_{2}:=D \epsilon \Delta J_{1}-D J_{1} \Delta \epsilon$

$$
=C^{2} P_{2}-2 C \epsilon\left(C+2 T^{2}\right) P_{1}-2 C\left(C+6 T^{2}\right) P^{2}+4 T\left[C^{2}+2 T\left(C+2 T^{2}\right) \epsilon^{2}\right] P .
$$

Hence, the invariants $J_{1}$ and $J_{2}$ have order 4 and 5 respectively. If $T=0$ then $J=-C^{2}$ is constant. In the generic case $C T \neq 0$, and in the light of (55) and analogous structure equations for $d J$, the invariant $J$ is non-constant and of order 3 . Explicit calculations relative to the metric form (4) show that

$$
\begin{aligned}
& \epsilon=C \mathrm{e}^{-2 T w} x \\
& J=-C^{2} \mathrm{e}^{-4 T w}(1+2 T F(u)), \\
& A:=\frac{\left(C J_{1}+4 T \epsilon J\right)^{2}}{J^{3}}=-\frac{\left(F^{\prime}(u)\right)^{2}}{(1+2 T F(u))^{3}} \\
& B:=\frac{C J_{2}+20 C J_{1} T^{2} \epsilon}{J^{2}}+\frac{48 T^{3} \epsilon^{2}}{J}=-\frac{F^{\prime \prime}(u)}{(1+2 T F(u))^{2}} .
\end{aligned}
$$


The latter two invariants have order 4 and 5 , respectively. The metric is classified by the functional relationship between these invariants. Observe that the maximal order condition is $B \neq 3 T A$.

If $C=0 \neq T$ we define dimensionless invariants of order 3,4 and 5 :

$$
p:=P / \epsilon^{2}, \quad p_{1}:=-P_{1} / \epsilon^{3}, \quad p_{2}:=P_{2} / \epsilon^{4} .
$$

Explicit calculations relative to (4) now give

$$
\begin{aligned}
& \epsilon=2 T^{2} \mathrm{e}^{-2 T w}, \quad p=2 x \\
& U:=p_{1}+\frac{3 p^{2}}{2}+2 T^{2}\left(p+\frac{T}{\epsilon^{2}}\right)=\frac{F(u)}{T^{2}}+\frac{1}{2 T^{3}}, \\
& V:=p_{2}+2(3 p+1) p_{1}+6 p^{3}+4 p\left(p+\frac{T}{\epsilon^{2}}\right)=-\frac{F^{\prime}(u)}{2 T^{4}} .
\end{aligned}
$$

Hence, as above, the metric is classified by the functional relationship between a fourth and a fifth order invariant. The maximal order condition is $V \neq 0$.

\section{Conclusions and discussion}

In this paper we have demonstrated that three-dimensional Lorentzian metrics may require fifth order differential invariants for their invariant classification. The class of maximal order metrics consists of a single, well-defined family of $\mathrm{CH}_{1}$ solutions governed by a unified set of structure equations. This echoes a similar result in four-dimensional Lorentzian geometry [18], although there the possibility of pseudo-stable geometries of maximal order was left open.

Previously, three-dimensional Lorentzian $\mathrm{CH}_{1}$ metrics were studied in detail by Bueken and Djoric [5]. They already proved proposition 15 and obtained the metrics covered by propositions 12 and 17, albeit not in closed form but up to solving partial differential equations. The coordinate forms in [5] are therefore less convenient for invariant classification and the discussion of the order, whereas our work was more directly related to Cartan invariants. Even though our focus here was on type $\mathrm{D}$ metrics of maximal order, the type $\mathrm{N}$, third order $\mathrm{CH}_{1}$ geometries from proposition 12 also constitute an interesting class governed by a well-defined set of structure equations. A closed form for these metrics can be derived along the same lines as in the type D case outlined above, but we do not pursue this here.

In [9] it was proved that the unique TMG solution of type $\mathrm{D}$ (dubbed type $\mathrm{D}_{s}$ there, cf table $\mathrm{C} 1$ of appendix $\mathrm{C}$ ) is the homogeneous, biaxially spacelike-squashed $\mathrm{AdS}_{3}$ metric family; this is the unique solution corresponding to the proof of proposition 16. Type D NMG solutions with constant scalar curvature were fully classified in [2] and are also homogeneous. Hence, the metrics of theorem 1 are not TMG nor NMG solutions. Therefore, our conclusion is that

\section{at most four covariant derivatives of the Riemann tensor are needed to invariant classify exact TMG and NMG solutions locally.}

In future work, we want to sharpen this result. Hereby, the technique we have followed in this paper to prove propositions $12-17$ not only provides a robust mechanism to invariantly characterize solutions, but also allows one to find new solutions, beyond the curvature homogeneity assumption. A first step, however, would be to classify all curvature homogeneous TMG and NMG solutions, in order to see whether the bound $q-1 \leqslant 3$ for the TMG and NMG gravitational theories is sharp. 
Finally, the same argument given for proposition 9 holds for Riemannian geometry as well. However, for Euclidean signature only the equivalent of type DZ curvature is possible and this suffices to rule out fourth order Riemannian metrics. However third order, three-dimensional order Riemannian metrics are possible. We will report on this fact elsewhere.

\section{Acknowledgments}

RM was supported by an NSERC discovery grant. He thanks the Mathematical Institute of Utrecht University for its hospitality during a research visit. LW was supported by a BOF research grant of Ghent University, an FWO mobility grant no V4.356.10N to Utrecht University and an Yggdrasil mobility grant no 211109 to University of Stavanger while parts of this work were performed. He thanks the Department of Mathematics and Statistics of Dalhousie University for its hospitality during a research stay.

\section{Appendix A. The three-dimensional formalism}

Several three-dimensional Newman-Penrose (NP)-like formalisms, with different symbol choices, have been proposed in the context of exact solutions to topologically massive gravity $[13,3]$. Our choice of symbols is close to [3], but differs slightly in the choice of normalization because we attempted to satisfy the following criteria:

- our three-dimensional formalism is obtainable as a straightforward reduction of the usual four-dimensional NP formalism [26, chapter 7];

- our rule for passing from vector to spinor indices is very simple and does not involve normalizing factors;

- the relation between the curvature spinor and the Ricci tensor takes a particularly simple form; see equation (A.24).

Let $(U, \varepsilon)$ be a two-dimensional symplectic, real vector space. The group of symplectic automorphisms is isomorphic to $\mathrm{SL}_{2} \mathbb{R}$. The vector space $V=\mathbf{S}^{2} U$ carries the natural structure of a Lorentzian inner product space with the inner product given by $\eta=-\varepsilon^{2}$. Henceforth, we regard $U$ as the space of spinors and $V$ as the space of vectors. The group $O(\eta)$ is isomorphic to $\mathrm{SO}(1,2)$; the group morphism $\mathrm{SL}_{2} \mathbb{R} \rightarrow \mathrm{SO}(1,2)$ gives the double cover of vectors by spinors.

To facilitate frame calculations, we introduce a normalized spinor dyad $\boldsymbol{o}, \boldsymbol{\iota}$ :

$$
\begin{aligned}
& \varepsilon_{01} \equiv \varepsilon_{A B} o^{A} \iota^{B} \equiv o_{A} \iota^{A}=-\iota_{A} o^{A} \equiv-\varepsilon_{10}=1, \\
& \varepsilon_{00} \equiv o_{A} o^{A}=0, \quad \varepsilon_{11} \equiv \iota_{A} \iota^{A}=0,
\end{aligned}
$$

where the dyad indices $A, B, \ldots$ take values 0 or 1 . Associated to this dyad, we define a null vector triad by

$$
\boldsymbol{e}_{0}=\boldsymbol{\ell}=\boldsymbol{o}^{2}, \quad \boldsymbol{e}_{1}=\boldsymbol{m}=\boldsymbol{o} \boldsymbol{\iota} \equiv \frac{1}{2}(\boldsymbol{o} \otimes \boldsymbol{\iota}+\boldsymbol{\iota} \otimes \boldsymbol{o}), \quad \boldsymbol{e}_{2}=\boldsymbol{n}=\boldsymbol{\iota}^{2},
$$

where the triad indices $a, b, c=0,1,2$ are doublets of symmetrized dyad indices:

$$
0 \mapsto(00), \quad 1 \mapsto(01), \quad 2 \mapsto(11) .
$$

In this way, we have

$$
\begin{aligned}
& \eta_{a b}=\eta_{\left(A_{1} A_{2}\right)\left(B_{1} B_{2}\right)}=-\frac{1}{2}\left(\varepsilon_{A_{1} B_{1}} \varepsilon_{A_{2} B_{2}}+\varepsilon_{A_{1} B_{2}} \varepsilon_{A_{2} B_{1}}\right), \\
& \eta_{02}=\eta_{20}=-1, \quad \eta_{11}=1 / 2,
\end{aligned}
$$

with all other components zero. Equivalently,

$$
\eta_{a b} \ell^{a} n^{b}=\ell_{a} n^{a}=-1, \quad m_{a} m^{a}=1 / 2,
$$


with all other inner products equal to zero.

Next, let $(M, g)$ be a three-dimensional Lorentzian manifold. A null triad at $x \in M$ is an isomorphism $(V, \eta) \rightarrow\left(T_{x} M, g_{x}\right)$. A moving $\eta$-frame is a null triad at every $x \in O$ for some open neighbourhood $O \subset M$. Equivalently, a null triad is a collection of vector fields $\boldsymbol{\ell}, \boldsymbol{m}, \boldsymbol{n}$ that satisfy the relations

$$
g(\boldsymbol{\ell}, \boldsymbol{n})=-1, \quad g(\boldsymbol{m}, \boldsymbol{m})=1 / 2,
$$

with all other inner products zero. In other words, taking $\left(\boldsymbol{e}_{0}, \boldsymbol{e}_{1}, \boldsymbol{e}_{2}\right)=(\boldsymbol{\ell}, \boldsymbol{m}, \boldsymbol{n})$ gives

$$
\left(g_{a b}\right)=\left(\eta_{a b}\right)=\left(\begin{array}{ccc}
0 & 0 & -1 \\
0 & 1 / 2 & 0 \\
-1 & 0 & 0
\end{array}\right), \quad\left(g^{a b}\right)=\left(\eta^{a b}\right)=\left(\begin{array}{ccc}
0 & 0 & -1 \\
0 & 2 & 0 \\
-1 & 0 & 0
\end{array}\right) .
$$

In introducing symbols for the connection scalars, we wish to adapt the notation of the familiar four-dimensional NP formalism. To do so, it is convenient to regard the manifold $M$ as a totally geodesic embedding (all geodesics in the submanifold are also geodesics of the surrounding manifold) $\phi: M \hookrightarrow \hat{M}$ in a four-dimensional Lorentzian manifold $(\hat{M}, \hat{g})$. This is equivalent to the condition that $M$ be autoparallel, i.e., that the covariant derivative operator is closed with respect to vector fields that are tangent to $M$ [16, chapter 7 , section 8].

Recall that a null tetrad framing on $\hat{M}$ is a basis of vector fields $\left(\hat{\boldsymbol{m}}, \hat{\boldsymbol{m}}^{*}, \hat{\boldsymbol{n}}, \hat{\boldsymbol{\ell}}\right)$ such that

$$
\hat{g}\left(\hat{\boldsymbol{m}}, \hat{\boldsymbol{m}}^{*}\right)=1, \quad \hat{g}(\hat{\boldsymbol{\ell}}, \hat{\boldsymbol{n}})=-1,
$$

with all other cross-products equal to zero. Here $\hat{\boldsymbol{\ell}}, \hat{\boldsymbol{n}}$ are real whereas $\hat{\boldsymbol{m}}, \hat{\boldsymbol{m}}^{*}$ are complex conjugates. We relate the null tetrad on $\hat{M}$ to the null triad on $M$ by setting

$$
\phi_{*} \ell=\hat{\boldsymbol{\ell}}, \quad \phi_{*} \boldsymbol{m}=\operatorname{Re} \hat{\boldsymbol{m}}=\left(\hat{\boldsymbol{m}}+\hat{\boldsymbol{m}}^{*}\right) / 2, \quad \phi_{*} \boldsymbol{n}=\hat{\boldsymbol{n}} .
$$

Let $\hat{\boldsymbol{\omega}}^{i}, i=1,2,3,4$ and $\hat{\boldsymbol{\Gamma}}_{i j}$ denote the dual coframe and the connection 1-form on $\hat{M}$. Let

$$
\tilde{\boldsymbol{\omega}}^{i}=\phi^{*} \hat{\boldsymbol{\omega}}^{i}, \quad \tilde{\boldsymbol{\Gamma}}_{i j}=\phi^{*} \hat{\boldsymbol{\Gamma}}_{i j}
$$

denote the corresponding pullbacks to $M$. Henceforth, we use a tilde decoration to denote the pullback of objects from $\hat{M}$ to $M$. The pullback imposes the condition:

$$
\tilde{\omega}^{1}=\tilde{\omega}^{2} .
$$

The embedding of $M$ into $\hat{M}$ induces an inclusion of the three-dimensional Lorentz group $\mathrm{SO}(1,2)$ into $\mathrm{SO}(1,3)$, the four-dimensional Lorentz group. The condition that $M$ be autoparallel is equivalent to the condition that the pull-back of the connection 1-form take values in the subalgebra $\mathfrak{s o}(1,2)$. This imposes the following conditions on the pullback of the connection 1-form:

$$
\tilde{\boldsymbol{\Gamma}}_{14}=\tilde{\boldsymbol{\Gamma}}_{24}, \quad \tilde{\boldsymbol{\Gamma}}_{23}=\tilde{\boldsymbol{\Gamma}}_{13}, \quad \tilde{\boldsymbol{\Gamma}}_{12}=0 .
$$

Using the notation of [26, section 7.2], the corresponding condition on the NP connection scalars is:

$$
\begin{aligned}
& \operatorname{Im} \tilde{\kappa}=\operatorname{Im} \tilde{\tau}=\operatorname{Im} \tilde{\epsilon}=\operatorname{Im} \tilde{\gamma}=\operatorname{Im} \tilde{\pi}=\operatorname{Im} \tilde{v}=0, \\
& \operatorname{Im}(\tilde{\sigma}+\tilde{\rho})=\operatorname{Im}(\tilde{\alpha}+\tilde{\beta})=\operatorname{Im}(\tilde{\lambda}+\tilde{\mu})=0 .
\end{aligned}
$$

Taking into account the difference in the ordering of the three-dimensional and the fourdimensional indices, we arrive at the following notation for the three-dimensional connection 1-form and scalars:

$$
\omega^{0}=\tilde{\omega}^{4}, \quad \omega^{1}=2 \tilde{\omega}^{1}=2 \tilde{\omega}^{2}, \quad \omega^{2}=\tilde{\omega}^{3}
$$




$$
\begin{aligned}
& \left(\boldsymbol{\Gamma}_{b}^{a}\right)=\left(\begin{array}{ccc}
\boldsymbol{\Gamma}_{02} & \boldsymbol{\Gamma}_{12} & 0 \\
-2 \boldsymbol{\Gamma}_{01} & 0 & 2 \boldsymbol{\Gamma}_{12} \\
0 & -\boldsymbol{\Gamma}_{01} & -\boldsymbol{\Gamma}_{02}
\end{array}\right), \\
& \boldsymbol{\Gamma}_{01}=-\tilde{\boldsymbol{\Gamma}}_{14}=\kappa \boldsymbol{\omega}^{0}+\sigma \boldsymbol{\omega}^{1}+\tau \boldsymbol{\omega}^{2}, \\
& \boldsymbol{\Gamma}_{02}=-\tilde{\boldsymbol{\Gamma}}_{34}=2\left(\epsilon \boldsymbol{\omega}^{0}+\alpha \boldsymbol{\omega}^{1}+\gamma \boldsymbol{\omega}^{2}\right), \\
& \boldsymbol{\Gamma}_{12}=\tilde{\boldsymbol{\Gamma}}_{23}=\pi \boldsymbol{\omega}^{0}+\lambda \boldsymbol{\omega}^{1}+v \boldsymbol{\omega}^{2} ; \\
& \kappa=\tilde{\kappa}, \quad \tau=\tilde{\tau}, \quad \sigma=(\tilde{\sigma}+\tilde{\rho}) / 2, \\
& \pi=\tilde{\pi}, \quad \nu=\tilde{v}, \quad \lambda=(\tilde{\lambda}+\tilde{\mu}) / 2, \\
& \epsilon=\tilde{\epsilon}, \quad \gamma=\tilde{\gamma}, \quad \alpha=(\tilde{\alpha}+\tilde{\beta}) / 2 .
\end{aligned}
$$

Writing

$$
D=\ell^{a} \nabla_{a}, \quad \delta=m^{a} \nabla_{a}, \quad \Delta=n^{a} \nabla_{a},
$$

we have by (A.5):

$$
D \tilde{\psi}=\phi^{*}(\hat{D} \hat{\psi}), \quad \delta \tilde{\psi}=\phi^{*}\left(\hat{\delta} \hat{\psi}+\hat{\delta}^{*} \hat{\psi}\right) / 2, \quad \Delta \tilde{\psi}=\phi^{*} \hat{\Delta} \hat{\psi},
$$

where $\hat{\psi}$ is a scalar defined on $\hat{M}$ and $\tilde{\psi}=\phi^{*} \hat{\psi}$ is its pullback to $M$. The three-dimensional commutator relations can now be expressed as

$$
\begin{aligned}
& D \delta-\delta D=(\pi-2 \alpha) D+2 \sigma \delta-\kappa \Delta, \\
& D \Delta-\Delta D=-2 \gamma D+2(\tau+\pi) \delta-2 \epsilon \Delta, \\
& \delta \Delta-\Delta \delta=-\nu D+2 \lambda \delta+(\tau-2 \alpha) \Delta .
\end{aligned}
$$

The above equations follow in a straightforward manner by applying symbol rules (A.14)(A.16), (A.18) to the usual four-dimensional commutator relations, as shown for example in equations (7.6a)- (7.6c) of [26].

The three-dimensional curvature tensor $R_{a b c d}$ decomposes into a curvature scalar

$$
R \equiv R_{a}^{a}, \quad R_{a b} \equiv R_{a c b}^{c},
$$

and a trace-free part

$$
S_{a b} \equiv R_{a b}-\frac{1}{3} R g_{a b}
$$

according to

$$
R_{a b c d}=\left(S_{a c} g_{b d}+S_{b d} g_{a c}-S_{b c} g_{a d}-S_{a d} g_{b c}\right)+\frac{1}{6} R\left(g_{a c} g_{b d}-g_{a d} g_{b c}\right) .
$$

The image of the natural inclusion $\mathbf{S}^{4} U \hookrightarrow \mathbf{S}^{2} V$ is the five-dimensional vector space of tracefree, symmetric tensors. Therefore, the trace-free part of a three-dimensional curvature tensor can be represented by means of a rank-4, symmetric curvature spinor:

$$
\Psi_{A B C D}=\left(\Psi_{0} \iota^{4}+4 \Psi_{1} \iota^{3} \boldsymbol{o}+6 \Psi_{2} \iota^{2} \boldsymbol{o}^{2}+4 \Psi_{3} \iota \boldsymbol{o}^{3}+\Psi_{4} \boldsymbol{o}^{4}\right)_{(A B C D)} .
$$

In this way, the definition of the curvature scalars $\Psi_{0}, \Psi_{1}, \Psi_{2}, \Psi_{3}, \Psi_{4}$ is formally identical to their four-dimensional counterparts; cf [26, equation (3.76)]. We obtain the following representation of the trace-free part of the Ricci tensor and the curvature-two form:

$$
\left(S_{a b}\right)=\left(\begin{array}{lll}
\Psi_{0} & \Psi_{1} & \Psi_{2} \\
\Psi_{1} & \Psi_{2} & \Psi_{3} \\
\Psi_{2} & \Psi_{3} & \Psi_{4}
\end{array}\right) ;
$$




$$
\begin{aligned}
& \left(\boldsymbol{\Omega}^{a}{ }_{b}\right)=\left(\begin{array}{ccc}
\boldsymbol{\Omega}_{02} & \boldsymbol{\Omega}_{12} & 0 \\
-2 \boldsymbol{\Omega}_{01} & 0 & 2 \boldsymbol{\Omega}_{12} \\
0 & -\boldsymbol{\Omega}_{01} & -\boldsymbol{\Omega}_{02}
\end{array}\right), \\
& \boldsymbol{\Omega}_{01}=\frac{1}{2} \Psi_{0} \boldsymbol{\omega}^{0} \wedge \boldsymbol{\omega}^{1}+\Psi_{1} \boldsymbol{\omega}^{0} \wedge \boldsymbol{\omega}^{2}+\left(\Psi_{2} / 2+R / 12\right) \boldsymbol{\omega}^{1} \wedge \boldsymbol{\omega}^{2}, \\
& \boldsymbol{\Omega}_{02}=\Psi_{1} \boldsymbol{\omega}^{0} \wedge \boldsymbol{\omega}^{1}+\left(2 \Psi_{2}-R / 6\right) \omega^{0} \wedge \omega^{2}+\Psi_{3} \boldsymbol{\omega}^{1} \wedge \omega^{2}, \\
& \boldsymbol{\Omega}_{12}=\left(\Psi_{2} / 2+R / 12\right) \boldsymbol{\omega}^{0} \wedge \omega^{1}+\Psi_{3} \boldsymbol{\omega}^{0} \wedge \omega^{2}+\frac{1}{2} \Psi_{4} \omega^{1} \wedge \omega^{2} .
\end{aligned}
$$

The three-dimensional curvature 2-form and curvature scalars are related to their fourdimensional counterparts as follows:

$$
\begin{aligned}
& \boldsymbol{\Omega}_{01}=-\tilde{\mathbf{\Omega}}_{14}, \quad \boldsymbol{\Omega}_{02}=-\tilde{\mathbf{\Omega}}_{34}, \quad \boldsymbol{\Omega}_{12}=\tilde{\mathbf{\Omega}}_{23}, \quad \tilde{\mathbf{\Omega}}_{12}=0 \\
& \Psi_{0}=\tilde{\Psi}_{0}+\tilde{\Phi}_{00}, \\
& \Psi_{1}=\tilde{\Psi}_{1}+\tilde{\Phi}_{01} \\
& \Psi_{2}=\tilde{\Psi}_{2}+\tilde{\Phi}_{02} / 3+2 / 3 \tilde{\Phi}_{11}, \\
& \Psi_{3}=\tilde{\Psi}_{3}+\tilde{\Phi}_{12} \\
& \Psi_{4}=\tilde{\Psi}_{4}+\tilde{\Phi}_{22}, \\
& R=\tilde{R} / 2+4 \tilde{\Phi}_{02}-4 \tilde{\Phi}_{11},
\end{aligned}
$$

with the right-hand sides of the above equations all real, as a consequence of equations (A.7) and (A.8).

The three-dimensional version of the NP equations, or equivalently Cartan's second structure equations, take the form shown below. Using equations (A.14)-(A.16), (A.18), (A.30)-(A.35), it is straightforward to convert the four-dimensional NP equations into their three-dimensional counterparts. For example, the NP equations (7.21a) and (7.21b) of [26] read

$$
\begin{aligned}
& D \rho-\delta^{*} \kappa=\rho^{2}+\sigma \sigma^{*}+\left(\epsilon+\epsilon^{*}\right) \rho-\kappa^{*} \tau-\kappa\left(3 \alpha+\beta^{*}-\pi\right)+\Phi_{00}, \\
& D \sigma-\delta \kappa=\left(\rho+\rho^{*}\right) \sigma+\left(3 \epsilon-\epsilon^{*}\right) \sigma-\left(\tau-\pi^{*}+\alpha^{*}+3 \beta\right) \kappa+\Psi_{0} .
\end{aligned}
$$

Note that all of the symbols in the above two equations should have hats, but we omit the decoration for the sake of simplicity. Taking the average of these two equations, pulling back and using (A.14)-(A.16), (A.18), (A.30)-(A.35) gives equation (A.36) below. The rest of the three-dimensional structure equations are obtained via the same reduction procedure.

$$
\begin{aligned}
& D \sigma-\delta \kappa=(\pi-4 \alpha-\tau) \kappa+2(\epsilon+\sigma) \sigma+\Psi_{0} / 2, \\
& D \tau-\Delta \kappa=-4 \gamma \kappa+2(\tau+\pi) \sigma+\Psi_{1}, \\
& D \alpha-\delta \epsilon=2(\sigma-\epsilon) \alpha+(\epsilon+\sigma) \pi-(\gamma+\lambda) \kappa+\Psi_{1} / 2, \\
& \delta \tau-\Delta \sigma=2(\lambda-\gamma) \sigma-\kappa \nu+\tau^{2}+\Psi_{2} / 2+R / 12, \\
& D \gamma-\Delta \epsilon=2(\tau+\pi) \alpha+\pi \tau-4 \gamma \epsilon-\kappa \nu+\Psi_{2}-R / 12, \\
& D \lambda-\delta \pi=2(\sigma-\epsilon) \lambda-\kappa \nu+\pi^{2}+\Psi_{2} / 2+R / 12, \\
& \delta \gamma-\Delta \alpha=2(\lambda-\gamma) \alpha+(\lambda+\gamma) \tau-(\epsilon+\sigma) \nu+\Psi_{3} / 2, \\
& D \nu-\Delta \pi=-4 \epsilon \nu+2(\tau+\pi) \lambda+\Psi_{3}, \\
& \delta \nu-\Delta \lambda=(\tau-4 \alpha-\pi) \nu+2(\gamma+\lambda) \lambda+\Psi_{4} / 2 .
\end{aligned}
$$


Likewise, the differential Bianchi equations are obtained by averaging the four-dimensional Bianchi equations and applying equations (A.14)-(A.16), (A.18), (A.30)-(A.35). They are:

$$
\begin{aligned}
& \begin{aligned}
& \Delta \Psi_{0} / 2-\delta \Psi_{1}+D\left(\Psi_{2} / 2+R / 12\right) \\
&=(2 \gamma-\lambda) \Psi_{0}+(\pi-2 \alpha-2 \tau) \Psi_{1}+3 \sigma \Psi_{2}-\kappa \Psi_{3} \\
& \Delta \Psi_{1} / 2-\delta\left(\Psi_{2}-R / 12\right)+D \Psi_{3} / 2 \\
&=\nu \Psi_{0} / 2+(\gamma-2 \lambda) \Psi_{1}+(3 / 2)(\pi-\tau) \Psi_{2}+(2 \sigma-\epsilon) \Psi_{3}-\kappa \Psi_{4} / 2
\end{aligned} \\
& \begin{aligned}
\Delta\left(\Psi_{2} / 2+R / 12\right) & -\delta \Psi_{3}+D \Psi_{4} / 2 \\
= & \nu \Psi_{1}-3 \lambda \Psi_{2}+(2 \pi+2 \alpha-\tau) \Psi_{3}+(\sigma-2 \epsilon) \Psi_{4}
\end{aligned}
\end{aligned}
$$

\section{Appendix B. Lorentz transformations}

There are three different types of three-dimensional Lorentz transformations: boosts, spins, and null rotations. Each such transformation has a simple description as a transformation of spinor space, i.e., as an element of $\mathrm{SL}_{2} \mathbb{R}$. Infinitesimally, boosts have non-zero, real eigenvalues, spins have imaginary eigenvalues, and null rotations have zero eigenvalues (in other words, an infinitesimal null rotation is a nilpotent transformation of spinor space). To facilitate calculations, we represent these transformations in a natural spinor dyad, and present their induced action on a suitable associated vector triad and on the corresponding connection and curvature scalars. Consistent with our philosophy of concordance between the threedimensional and four-dimensional formalisms, all of the above equations are straightforward reductions of the four-dimensional transformation laws; cf [27, appendix B].

A boost transformation corresponds to a real-diagonalizable element of $\mathrm{SL}_{2} \mathbb{R}$. The corresponding spinor and vector actions are

$$
\begin{aligned}
& \boldsymbol{o}^{\prime}=a^{1 / 2} \boldsymbol{o}, \quad \boldsymbol{\iota}^{\prime}=a^{-1 / 2} \boldsymbol{\iota}, \quad a>0, \\
& \boldsymbol{\ell}^{\prime}=a \boldsymbol{\ell}, \quad \boldsymbol{m}^{\prime}=\boldsymbol{m}, \quad \boldsymbol{n}^{\prime}=a^{-1} \boldsymbol{n} .
\end{aligned}
$$

Boost transformations can also be realized as the one-dimensional group of symmetries of the type D curvature spinor; cf line 6 of table C1. The associated connection and curvature transformation laws are shown below.

$$
\begin{aligned}
\tau^{\prime} & =\tau, \\
\pi^{\prime} & =\pi, \\
\sigma^{\prime} & =a \sigma, \\
\lambda^{\prime} & =a^{-1} \lambda, \\
\kappa^{\prime} & =a^{2} \kappa, \\
\nu^{\prime} & =a^{-2} \nu, \\
\epsilon^{\prime} & =a \epsilon+D a / 2, \\
\alpha^{\prime} & =\alpha+a^{-1} \delta a / 2, \\
\gamma^{\prime} & =a^{-1} \gamma+a^{-2} \Delta a / 2, \\
\Psi_{0}^{\prime} & =a^{2} \Psi_{0},
\end{aligned}
$$




$$
\begin{aligned}
& \Psi_{1}^{\prime}=a \Psi_{1}, \\
& \Psi_{2}^{\prime}=\Psi_{2}, \\
& \Psi_{3}^{\prime}=a^{-1} \Psi_{3}, \\
& \Psi_{4}^{\prime}=a^{-2} \Psi_{4} .
\end{aligned}
$$

A null rotation corresponds to a unipotent, non-diagonalizable element of $\mathrm{SL}_{2} \mathbb{R}$. The corresponding spinor and vector actions are

$$
\begin{array}{ll}
\boldsymbol{o}^{\prime}=\boldsymbol{o}, & \boldsymbol{\iota}^{\prime}=\boldsymbol{\imath}+x \boldsymbol{o}, \\
\boldsymbol{\ell}^{\prime}=\boldsymbol{\ell}, & \boldsymbol{m}^{\prime}=\boldsymbol{m}+x \boldsymbol{\ell}, \quad \boldsymbol{n}^{\prime}=\boldsymbol{n}+2 x \boldsymbol{m}+x^{2} \boldsymbol{\ell} .
\end{array}
$$

Null rotations can also be realized as the one-dimensional group of symmetries of the type $\mathrm{N}$ curvature spinor; cf line 9 of table $\mathrm{C} 1$. The associated transformation laws for the connection and curvature scalars are shown below.

$\kappa^{\prime}=\kappa$,

$\sigma^{\prime}=\sigma+x \kappa$,

$\epsilon^{\prime}=\epsilon+x \kappa$,

$\tau^{\prime}=\tau+2 x \sigma+x^{2} \kappa$,

$\alpha^{\prime}=\alpha+x(\epsilon+\sigma)+x^{2} \kappa$,

$\gamma^{\prime}=\gamma+x(2 \alpha+\tau)+x^{2}(\epsilon+2 \sigma)+x^{3} \kappa$,

$\pi^{\prime}=\pi+D x+2 x \epsilon+x^{2} \kappa$,

$\lambda^{\prime}=\lambda+\delta x+x(2 \alpha+\pi+D x)+x^{2}(2 \epsilon+\sigma)+x^{3} \kappa$,

$v^{\prime}=v+\Delta x+2 x(\gamma+\lambda+\delta x)+x^{2}(4 \alpha+\tau+\pi+D x)+2 x^{3}(\epsilon+\sigma)+x^{4} \kappa$,

$\Psi_{0}^{\prime}=\Psi_{0}$,

$\Psi_{1}^{\prime}=\Psi_{1}+x \Psi_{0}$,

$\Psi_{2}^{\prime}=\Psi_{2}+2 x \Psi_{1}+x^{2} \Psi_{0}$,

$\Psi_{3}^{\prime}=\Psi_{3}+3 x \Psi_{2}+3 x^{2} \Psi_{1}+x^{3} \Psi_{0}$,

$\Psi_{4}^{\prime}=\Psi_{4}+4 x \Psi_{3}+6 x^{2} \Psi_{2}+4 x^{3} \Psi_{1}+x^{4} \Psi_{0}$.

A spin transformation corresponds to an element of $\mathrm{SL}_{2} \mathbb{R}$ with imaginary eigenvalues. As such, we have

$$
\begin{aligned}
& \boldsymbol{o}^{\prime} \pm i \boldsymbol{\iota}^{\prime}=\mathrm{e}^{\mp i t / 2}(\boldsymbol{o} \pm i \boldsymbol{\iota}), \\
& (\boldsymbol{\ell}+\boldsymbol{n})^{\prime}=\mathrm{lhs}, \\
& (\boldsymbol{\ell}-\boldsymbol{n} \pm 2 i \boldsymbol{m})^{\prime}=\mathrm{e}^{\mp i t} \mathrm{hhs} .
\end{aligned}
$$

Spin transformations can also be realized as the one-parameter group of symmetries of the type DZ curvature spinor; cf line 7 of table $\mathrm{C} 1$. The associated connection and curvature 
transformation laws are shown below.

$$
\begin{aligned}
& (\gamma+\sigma-\epsilon-\lambda)^{\prime}=\mathrm{lhs}, \\
& (4 \alpha+\kappa-\pi+v-\tau)^{\prime}=\mathrm{lhs}, \\
& (2(\gamma+\epsilon) \pm \mathrm{i}(\kappa-\pi+\tau-v))^{\prime}=\mathrm{e}^{ \pm \mathrm{i} t} \mathrm{lhs}, \\
& (4 \alpha+\pi-\kappa+\tau-v+ \pm 2 \mathrm{i}(\epsilon-\gamma+\sigma-\lambda))=\mathrm{e}^{ \pm 2 \mathrm{it}} \mathrm{lhs}, \\
& (\lambda+\sigma-\gamma-\epsilon+ \pm \mathrm{i}(\pi-\tau))^{\prime}=\mathrm{e}^{ \pm \mathrm{i} t}(\mathrm{lhs}-\delta t \mp(\mathrm{i} / 2)(D t-\Delta t)), \\
& (\kappa+\pi+v+\tau)^{\prime}=\mathrm{lhs}-(D t+\Delta t) \\
& \left(\Psi_{0}+2 \Psi_{2}+\Psi_{4}\right)^{\prime}=\mathrm{lhs}, \\
& \left(\Psi_{0}-\Psi_{4} \pm 2 \mathrm{i}\left(\Psi_{1}+\Psi_{3}\right)\right)^{\prime}=\mathrm{e}^{\mp \mathrm{it}} 1 \mathrm{hs}, \\
& \left(\Psi_{0}-6 \Psi_{2}+\Psi_{4} \pm 4 \mathrm{i}\left(\Psi_{1}-\Psi_{3}\right)\right)^{\prime}=\mathrm{e}^{\mp 2 \mathrm{it}} \text { lhs. }
\end{aligned}
$$

Finally, there are a number of discrete Lorentz transformations that lie outside the connected component of the identity in $O(\eta)$. Given a null frame $(\boldsymbol{\ell}, \boldsymbol{m}, \boldsymbol{n})$ we define

$$
\mathbf{t} \equiv \frac{1}{\sqrt{2}}(\boldsymbol{\ell}+\boldsymbol{n}), \quad \mathbf{x} \equiv \frac{1}{2}(\boldsymbol{\ell}-\boldsymbol{n}) .
$$

The transformation laws of the connection and curvature scalars under reflection of the vectors of the orthonormal triad $(\mathbf{t}, \mathbf{m}, \mathbf{x})$ are also relevant for our purposes and are given below.

Reflection of $\mathbf{t}$ ('time reversal'):

$$
\begin{aligned}
& \mathbf{t} \mapsto-\mathbf{t} \Leftrightarrow \boldsymbol{\ell} \mapsto-\boldsymbol{\ell}, \boldsymbol{n} \mapsto-\boldsymbol{n}: \\
& \kappa, \tau, \alpha, \pi, v \text { invariant, } \sigma, \epsilon, \gamma, \lambda \text { change sign, } \\
& \Psi_{0}, \Psi_{2}, \Psi_{4} \text { invariant, } \Psi_{1}, \Psi_{3} \text { change sign. }
\end{aligned}
$$

Reflection of $\mathbf{m}$ :

$$
\begin{aligned}
& \mathbf{m} \mapsto-\mathbf{m}: \\
& \kappa, \tau, \alpha, \pi, \nu \text { change sign, } \sigma, \epsilon, \gamma, \lambda \text { invariant, } \\
& \Psi_{0}, \Psi_{2}, \Psi_{4} \text { invariant, } \Psi_{1}, \Psi_{3} \text { change sign. }
\end{aligned}
$$

Reflection of $\mathbf{x}$ :

$$
\begin{aligned}
& \mathbf{x} \mapsto-\mathbf{x} \Leftrightarrow \quad \boldsymbol{l} \leftrightarrow \boldsymbol{n}: \\
& \kappa \leftrightarrow-v, \quad \sigma \leftrightarrow-\lambda, \quad \tau \leftrightarrow-\pi, \quad \epsilon \leftrightarrow-\gamma, \quad \alpha^{\prime}=-\alpha, \\
& \Psi_{0} \leftrightarrow \Psi_{4}, \quad \Psi_{1} \leftrightarrow \Psi_{3}, \quad \Psi_{2}^{\prime}=\Psi_{2} .
\end{aligned}
$$

\section{Appendix C. Petrov-Penrose classification of the three-dimensional Ricci tensor}

Let $\boldsymbol{\ell}, \boldsymbol{m}, \boldsymbol{n}$ a null vector triad for which $\Psi_{4} \neq 0$. We introduce the three-dimensional analogue of the Petrov-Penrose classification in terms of the root configurations of the real quartic

$$
\Psi_{0}(z)=\Psi_{0}+4 \Psi_{1} z+6 \Psi_{2} z^{2}+4 \Psi_{3} z^{3}+\Psi_{4} z^{4} .
$$

We note that this classification forms a special case of the general null alignment classification for tensors in arbitrary dimensions [17], applied here to the three-dimensional trace-free Ricci tensor $S_{a b}$. Hence, in addition to the analogues of Petrov types I, II, D, III, N (where there are four real solutions $z$ ) and type $\mathrm{O}$, we have to account for the possibility that some or all of 
Table C1. The three-dimensional Petrov-Segre type.

\begin{tabular}{lllll}
\hline Petrov type & Segre type & {$[9]$} & Normalization & $s_{0}$ \\
\hline I & $\{11,1\}$ & I $_{\mathbb{R}}$ & $\Psi_{1}=\Psi_{3}=0, \Psi_{0}=\Psi_{4}, 3 \Psi_{2} / \Psi_{0}<-1$ & 0 \\
IZ & $\{1 z \bar{z}\}$ & I $_{\mathbb{C}}$ & $\Psi_{1}=\Psi_{3}=0, \Psi_{0}=-\Psi_{4} \neq 0$, & 0 \\
IZZ & $\{11,1\}$ & I $_{\mathbb{R}}$ & $\Psi_{1}=\Psi_{3}=0, \Psi_{0}=\Psi_{4}, 3 \Psi_{2} / \Psi_{0}>1$ & 0 \\
II & $\{21\}$ & II & $\Psi_{1}=\Psi_{3}=\Psi_{4}=0, \Psi_{2} / \Psi_{0}<0$ & 0 \\
IIZ & $\{21\}$ & II & $\Psi_{1}=\Psi_{3}=\Psi_{4}=0, \Psi_{2} / \Psi_{0}>0$ & 0 \\
D & $\{(11), 1\}$ & D $_{s}$ & $\Psi_{0}=\Psi_{1}=\Psi_{3}=\Psi_{4}=0, \Psi_{2} \neq 0$ & 1 \\
DZ & $\{1(1,1)\}$ & D $_{t}$ & $\Psi_{1}=\Psi_{3}=0, \Psi_{0}=\Psi_{4}=3 \Psi_{2} \neq 0$ & 1 \\
III & $\{3\}$ & III & $\Psi_{0}=\Psi_{1}=\Psi_{2}=\Psi_{4}=0, \Psi_{3}=1$ & 0 \\
N & $\{(21)\}$ & N & $\Psi_{0}=\Psi_{1}=\Psi_{2}=\Psi_{3}=0, \Psi_{4}= \pm 1$ & 1 \\
O & $\{(11,1)\}$ & O & $\Psi_{0}=\Psi_{1}=\Psi_{2}=\Psi_{3}=\Psi_{4}=0$ & 3 \\
\hline
\end{tabular}

the roots of $\Psi(z)$ are complex. We will denote these additional root configurations as Petrov types IZ (two different real roots, two complex roots), IZZ (four complex roots), IIZ (double real root, two complex roots), and DZ (the double roots are complex conjugate).

Table C1 summarizes the three-dimensional Petrov types, corresponding Segre types of the trace-free Ricci operator $S^{a}{ }_{b}$, the notation introduced in [9] for the latter, and possible normalized forms; the last column shows the dimension $s_{0}$ of the corresponding automorphism group. An alternate normalized form for type IZZ is given by

$$
\Psi_{1}=\Psi_{3}=0, \Psi_{0}=\Psi_{4}, 3\left|\Psi_{2} / \Psi_{0}\right|<1,
$$

but it is related to the form in the table by a Lorentz transformation. Analogously, a Lorentzequivalent type $\mathrm{D}$ canonical form is

$$
\Psi_{1}=\Psi_{3}=0, \Psi_{0}=\Psi_{4}=-3 \Psi_{2} .
$$

Note that the Ricci-Petrov classification based on null alignment refines the Ricci-Segre type classification [13]. The distinction between Petrov types I and IZZ is the order of the timelike eigenvalue, relative to the spacelike eigenvalues. Regarding Segre type $\{21\}$, the spacelike or timelike character of the vector $S_{a b} l^{b}$, where the null vector $\ell$ lies in the two-dimensional generalized eigenspace but is not an eigenvector, distinguishes between Petrov types II and IIZ. Also note that Petrov type $\mathrm{O}$ describes a constant curvature space.

\section{Appendix D. $\mathrm{CH}_{1}$ structure equations}

This appendix is devoted to an analysis of the algebraic data and the structure equations that underly curvature homogeneous geometries. In what follows a crucial, albeit technical, innovation allows us to simplify the form of higher order Cartan invariants by replacing them with certain connection scalars. The general theory is detailed in [19]. For the sake of concreteness we limit the discussion to the case of $\mathrm{CH}_{1}$ geometries. We begin by recalling some preliminary notation and theory, and then turn to the description of $\mathrm{CH}_{1}$ data and structure equations, which we call a $\mathrm{CH}_{1}$ configuration.

Let $\boldsymbol{e}_{a}, a=1, \ldots, n$ be a basis of $V$, and $\boldsymbol{A}_{\alpha}, \alpha=1, \ldots, n(n-1) / 2$ a basis of $\mathfrak{o}(\eta)$. Let $A_{b \alpha}^{a}$ denote the matrix components of $\boldsymbol{A}_{\alpha}$; i.e.,

$$
\boldsymbol{A}_{\alpha} \cdot \boldsymbol{e}_{b}=A^{a}{ }_{b \alpha} \boldsymbol{e}_{a} .
$$

Let $C^{\alpha}{ }_{\beta \gamma}$ be the corresponding structure constants:

$$
\left[\boldsymbol{A}_{\beta}, \boldsymbol{A}_{\gamma}\right]=C_{\beta \gamma}^{\alpha} \boldsymbol{A}_{\alpha}, \quad A^{a}{ }_{e \beta} A_{b \gamma}^{e}-A^{a}{ }_{e \gamma} A_{b \beta}^{e}=A_{b \alpha}^{a} C_{\beta \gamma}^{\alpha} .
$$


Let $\omega^{a}$ be an $\eta$-orthogonal coframe, $\Gamma^{\alpha}, \Omega^{\alpha}$ the corresponding connection 1-form and curvature 2-form, respectively. The latter are determined by the first and second structure equations:

$$
\begin{aligned}
\mathrm{d} \omega^{a} & =-A_{b \alpha}^{a} \Gamma^{\alpha} \wedge \omega^{b}, \\
\mathrm{~d} \Gamma^{\alpha} & =-\frac{1}{2} C^{\alpha}{ }_{\beta \gamma} \Gamma^{\beta} \wedge \Gamma^{\gamma}+\Omega^{\alpha},
\end{aligned}
$$

where $\Gamma_{a}^{\alpha}, R_{a b c d}$ are the connection and curvature components, respectively:

$$
\begin{aligned}
& \Gamma^{\alpha}=\Gamma_{a}^{\alpha} \omega^{a}, \\
& \Omega^{\alpha}=\frac{1}{2} R_{c d}^{\alpha} \omega^{c} \wedge \omega^{d}, \quad R_{a b c d}=A_{a b \alpha} R_{c d}^{\alpha} .
\end{aligned}
$$

The exterior derivative gives the algebraic and differential Bianchi relations:

$$
\begin{aligned}
& A_{b \alpha}^{a} \Omega^{\alpha} \wedge \omega^{b}=0, \\
& \mathrm{~d} \Omega^{\alpha}=\frac{1}{2} C^{\alpha}{ }_{\beta \gamma} \Omega^{\beta} \wedge \Gamma^{\gamma} .
\end{aligned}
$$

In appendix $\mathrm{A}$ we introduced a convenient formalism that assigns specific symbols to the $\Gamma_{a}^{\alpha}, R_{a b c d}$ when $n=3$. Our three-dimensional formalism is a suitable reduction of the wellknown four-dimensional NP formalism. In this reduced, three-dimensional formalism, the first structure equations (D.1) correspond to the commutator relations (A.19)-(A.21); the second structure equations correspond to reduced NP equations (A.36)-(A.44). The component versions of the Bianchi relations are given by (A.45)-(A.47). The details of the formalism and of the reduction from 4 to 3 dimensions are given in appendix A.

Next, suppose that the $\mathrm{CH}_{1}$ condition holds and let $\omega^{a}$ be a curvature normalized $\eta$ orthogonal coframe. By the $t_{1}=0$ assumption,

$$
R_{a b c d}=\tilde{R}_{a b c d}, \quad R_{a b c d ; e}=\tilde{R}_{a b c d e},
$$

where the right hand sides denote arrays of constants. Let $G_{0} \subset O(\eta)$ be the automorphisms of $\tilde{R}_{a b c d}$ and $G_{1} \subset G_{0}$ the automorphisms of $\tilde{R}_{a b c d e}$. Hence, (D.7) fixes the choice of coframe up to a $G_{1}$ gauge transformation. Set

$$
\mathfrak{g}_{-1}:=\mathfrak{o}(\eta), \quad s_{-1}=\operatorname{dim} \mathfrak{g}_{-1}=n(n-1) / 2 .
$$

Let $\mathfrak{g}_{0}, \mathfrak{g}_{1}$ denote the Lie algebra of $G_{0}, G_{1}$ respectively. Introduce an adapted basis of $\mathfrak{g}_{1} \subset \mathfrak{g}_{0} \subset \mathfrak{g}_{-1}$ consisting of

$$
\left(\boldsymbol{A}_{\xi}, \boldsymbol{A}_{\lambda}, \boldsymbol{A}_{\rho}\right), \quad \xi=1, \ldots s_{1}, \lambda=s_{1}+1, \ldots s_{0}, \rho=s_{0}+1, \ldots, s_{-1},
$$

where the $\boldsymbol{A}_{\xi}$ are a basis of $\mathfrak{g}_{1}$, the $\boldsymbol{A}_{\lambda}$ are a basis of $\mathfrak{g}_{0} / \mathfrak{g}_{1}$ and the $\boldsymbol{A}_{\rho}$ are a basis of $\mathfrak{g}_{-1} / \mathfrak{g}_{0}$. By the usual definition of the covariant derivative one has

$$
R_{a b c d ; e}=R_{a b c d, e}+\Gamma_{e}^{\alpha}\left(A_{\alpha} \cdot R\right)_{a b c d},
$$

where for a rank $k$ tensor $T_{a_{1} \ldots a_{k}}$ the notation

$$
(\boldsymbol{A} \cdot T)_{a_{1} \ldots a_{k}}=-\sum_{i=1}^{k} A^{b}{ }_{a_{i}} T_{a_{1} \cdots \widehat{a}_{i} b \cdots a_{k}}, \quad \boldsymbol{A} \in \operatorname{End}(V)
$$

denotes the infinitesimal action of a linear transformation on a covariant tensor.

Since the $R_{a b c d}$ are constant and since $\mathfrak{g}_{0}$ is the annihilator of $\tilde{R}_{a b c d}$, (D.8) becomes

$$
\tilde{R}_{a b c d e}=\Gamma_{e}^{\rho}\left(\boldsymbol{A}_{\rho} \cdot \tilde{R}\right)_{a b c d} .
$$

Equation (D.9) describes a linear system in $\Gamma^{\rho}{ }_{e}$ with maximal rank. Hence, $\Gamma^{\rho}{ }_{e}=\tilde{\Gamma}^{\rho}{ }_{e}$, where the latter are constants rationally dependent on $\tilde{R}_{a b c d}, \tilde{R}_{a b c d e}$. Therefore, a $\mathrm{CH}_{1}$ geometry is 
determined by constants $\tilde{R}_{a b}^{\alpha}=-\tilde{R}_{b a}^{\alpha}$ and constants $\tilde{\Gamma}^{\rho}{ }_{a}$ such that the following relations hold, relative to a normalized $\eta$-orthogonal coframe:

$$
\begin{aligned}
& \tilde{R}_{a b c d}=A_{a b \alpha} \tilde{R}_{c d}^{\alpha}, \\
& \tilde{R}_{a b c d e}=\tilde{\Gamma}_{a}^{\rho}{ }_{a}\left(\boldsymbol{A}_{\rho} \cdot \tilde{R}\right)_{a b c d} .
\end{aligned}
$$

Moreover, the Bianchi relations (D.5), (D.6) impose the following linear, respectively, bilinear constraints on the above constants:

$$
\begin{aligned}
& \tilde{R}_{[b c}^{\alpha} A^{a}{ }_{d] \alpha}=0, \\
& \left(\boldsymbol{A}_{\rho} \cdot \tilde{R}\right)^{\alpha}{ }_{[a b} \tilde{\Gamma}_{c]}^{\rho}=0 .
\end{aligned}
$$

Similar to (D.9), the second order derivative of curvature is given by

$$
R_{a b c d ; e f}=\tilde{\Gamma}_{f}^{\rho}\left(\boldsymbol{A}_{\rho} \cdot \tilde{R}\right)_{a b c d e}+\Gamma_{f}^{\lambda}\left(\boldsymbol{A}_{\lambda} \cdot \tilde{R}\right)_{a b c d e},
$$

relative to a normalized coframe. Since the residual frame freedom is $G_{1}$, the scalars $\Gamma^{\lambda}{ }_{a}$ obey an algebraic, $G_{1}$-transformation law. The second structure equations (D.2) impose the following linear constraints on these scalars:

$$
\tilde{R}_{a b}^{\rho}=C^{\rho}{ }_{\rho_{1} \rho_{2}} \tilde{\Gamma}^{\rho_{1}}{ }_{a} \tilde{\Gamma}^{\rho_{2}}{ }_{b}-2 \tilde{\Gamma}^{\rho}{ }_{c} \tilde{\Gamma}^{\rho_{1}}{ }_{[a} A^{c}{ }_{b] \rho_{1}}-2\left(\boldsymbol{A}_{\lambda} \cdot \tilde{\Gamma}\right)^{\rho}{ }_{[a} \Gamma_{b]}^{\lambda},
$$

where $\rho_{1}, \rho_{2}=s_{0}+1, \ldots, s_{-1}$ have the same range as $\rho$, and the following differential relations:

$$
\Gamma_{[a, b]}^{\lambda}=\left(\boldsymbol{A}_{\xi} \cdot \Gamma\right)_{b}^{\lambda} \Gamma_{a}^{\xi}-\frac{1}{2} \Upsilon_{a b}^{\lambda},
$$

where

$$
\begin{aligned}
\Upsilon^{\lambda}{ }_{a b}:=\tilde{R}_{a b}^{\lambda} & -C^{\lambda}{ }_{\rho_{1} \rho_{2}} \tilde{\Gamma}^{\rho_{1}}{ }_{a} \tilde{\Gamma}^{\rho_{2}}{ }_{b}+2 \Gamma^{\lambda}{ }_{c} \tilde{\Gamma}^{\rho_{1}}{ }_{[a} A^{c}{ }_{b] \rho_{1}}-2 \Gamma^{\lambda_{1}}{ }_{a} \tilde{\Gamma}^{\rho_{1}}{ }_{b} C^{\lambda}{ }_{\lambda_{1} \rho_{1}} \\
& +2 \Gamma^{\lambda}{ }_{c} \Gamma^{\lambda_{1}}{ }_{[a} A^{c}{ }_{b] \lambda_{1}}-C^{\lambda}{ }_{\lambda_{1} \lambda_{2}} \Gamma^{\lambda_{1}}{ }_{a} \Gamma^{\lambda_{2}}{ }_{b},
\end{aligned}
$$

and where $\lambda_{1}, \lambda_{2}=s_{1}+1, \ldots s_{0}$ have the same range as $\lambda$. We will refer to constants $\tilde{R}_{a b}^{\alpha}, \tilde{\Gamma}_{a}^{\rho}$ and scalars $\Gamma_{a}^{\lambda}$ together with constraints (D.12)-(D.13) and (D.15)-(D.17) as a $\mathrm{CH}_{1}$ configuration [19].

It is well known that the $O(\eta)$ metric equivalence problem has trivial essential torsion [20, section 12]. However, if we reduce the structure group to $G_{0} \subset O(\eta)$ by means of curvature normalization, we obtain the following reduced first structure equations:

where

$$
\mathrm{d} \hat{\omega}^{a}=-\sum_{\xi=1}^{s_{0}} A_{b \xi}^{a} \hat{\Gamma}^{\xi} \wedge \hat{\omega}^{b}+\sum_{\rho} A_{b \rho}^{a} \hat{\Gamma}_{b}^{\rho} \hat{\omega}^{a} \wedge \hat{\omega}^{b}
$$

$$
\hat{\boldsymbol{\omega}}=X \boldsymbol{\omega}, \quad \hat{\Gamma}=X \Gamma X^{-1}-\mathrm{d} X X^{-1}, \quad X \in G_{0},
$$

are the $G_{0}$-lifted 1-forms. The scalars $\hat{\Gamma}^{\rho}{ }_{a}$ are well defined because the Maurer-Cartan term $\mathrm{d} X X^{-1}$ takes values in $\mathfrak{g}_{0}$. Consequently, the scalars $\Gamma^{\rho}{ }_{a}$ have a $G_{0}$-transformation law that does not depend on $\mathrm{d} X$, and therefore constitute the essential torsion for the 1 st iteration of the equivalence method. Thus, the scalars $\Gamma^{\rho}{ }_{a}$ can be interpreted as the essential torsion arising from the reduced $G_{0}$-equivalence problem and the $\Gamma^{\lambda}{ }_{a}$ as essential torsion in the next iteration of the $G_{1}$-equivalence problem. Therefore, we refer to the former as first order torsion, and to the latter as second order torsion.

By virtue of (D.9), normalizing the $\Gamma^{\rho}{ }_{a}$ is equivalent to normalizing $R_{a b c d ; e}$. The first order normalization reduces the structure group to $G_{1} \subset G_{0}$. If we suppose that the $\mathrm{CH}_{1}$ property holds, then the resulting invariants are the constants $\tilde{\Gamma}_{a}^{\rho}$. The scalars $\Gamma_{a}^{\lambda}$ are the essential torsion of the second iteration of the equivalence method. By virtue of (D.9), (D.14), the second order Cartan invariants are functions of the zeroth order Cartan invariants $R^{\alpha}{ }_{a b}$ and the first and second order torsion scalars $\tilde{\Gamma}_{a}^{\rho}, \Gamma_{a}^{\lambda}$. Inversely, because of (D.12), $\tilde{R}_{a b}^{\alpha}$ is linearly dependent on $\tilde{R}_{a b c d}$, while (D.11) and (D.14) can be solved to give $\tilde{\Gamma}^{\rho}{ }_{a}$ as functions of $\tilde{R}_{a b c d}, \tilde{R}_{a b c d e}$ and $\Gamma_{a}^{\lambda}$ as a function of $\tilde{R}_{a b c d}, \tilde{R}_{a b c d e}, R_{a b c d ; e f}$. 


\section{References}

[1] Ahmedov H and Aliev A N 2012 Type N spacetimes as solutions of extended new massive gravity Phys. Lett. B 711 117-21

[2] Ahmedov H and Aliev A N 2011 Type D solutions of 3D new massive gravity Phys. Rev. D 83084032

[3] Aliev A N and Nutku Y 1995 Spinor formulation of topologically massive gravity Class. Quantum Grav. 122913

[4] Boeckx E, Kowalski O and Vanhecke L 1996 Riemannian manifolds of Conullity Two (River Edge, NJ: World Scientific)

[5] Bueken P and Djorić M 2000 Three-dimensional Lorentz metrics and curvature homogeneity of order one Ann. Glob. Anal. Geom. 18 85-103

[6] Bryant R 2011 Cartan's generalization of Lie's third theorem CRM Workshop on Moving Frames (Montreal)

[7] Bryant R 2001 Bochner-Kähler metrics J. Am. Math. Soc. 14 623-715

[8] Cartan E 1946 Leçons sur la Géométrie des Espaces de Riemann (Paris: Gauthier-Villars)

[9] Chow D D K, Pope C N and Sezgin E 2010 Classification of solutions in topologically massive gravity Class. Quantum Grav. 27105001

[10] Collins J M and d'Inverno R A 1993 The Karlhede classification of type-D non-vacuum spacetimes Class. Quantum Grav. $10343-51$

[11] Fernandes R and Struchiner I 2007 Lie algebroids and classification problems in geometry arXiv:0712.3198

[12] Gilkey P 2007 The Geometry of Curvature Homogeneous Pseudo-Riemannian Manifolds (Cambridge, UK: Imperial College Press)

[13] Hall G S, Morgan T and Perjés Z 1987 Three-dimensional space-times Gen. Rel. Grav. 191137

[14] MacCallum M A H and Åman J E 1986 Algebraically independent nth derivatives of the Riemann curvature spinor in a general spacetime Class. Quantum Grav. 3 1133-41

[15] Karlhede A 1980 A review of the geometrical equivalence of metrics in general relativity Gen. Rel. Grav. 12 693-707

[16] Kobayashi S and Nomizu K 2009 Foundations of Differential Geometry vol 2 (Hoboken, NJ: Wiley)

[17] Milson R, Coley A, Pravda V and Pravdová A 2005 Alignment and algebraically special tensors in Lorentzian geometry Int. J. Geom. Methods Mod. Phys. 2 41-61

[18] Milson R and Pelavas N 2008 The type N Karlhede bound is sharp Class. Quantum Grav. 25012001

[19] Milson R and Pelavas N 2009 The curvature homogeneity bound for Lorentzian four-manifolds Int. J. Geom. Methods Mod. Phys. 6 99-127

[20] Olver P 1995 Equivalence, Invariants and Symmetry (Cambridge, UK: Cambridge University Press)

[21] Ozsváth I, Robinson I and Rózga K 1985 Plane-fronted gravitational and electromagnetic waves in spaces with cosmological constant J. Math. Phys. 1755-61

[22] Gardner R 1989 The Method of Equivalence and Its Applications (Philadelphia, PA: SIAM)

[23] Machado Ramos M P and Vickers J A G 1996 Invariant differential operators and the Karlhede classification of type N vacuum solutions Class. Quantum Grav. 13 1589-99

[24] Singer I M 1960 Infinitesimally homogeneous spaces Commun. Pure Appl. Math. 13 685-97

[25] Sousa F C, Fonseca J B and Romero C 2008 Equivalence of three-dimensional spacetimes Class. Quantum Grav. 25035007

[26] Stephani H, Kramer D, MacCallum M, Hoenselaers C and Herlt E 2003 Exact solutions of Einstein's field equations (Cambridge, UK: Cambridge University Press)

[27] Stewart J 1991 Advanced General Relativity (Cambridge, UK: Cambridge University Press)

[28] Wolf J 2011 Spaces of Constant Curvature 6th edn (Providence, RI: American Mathematical Society)

[29] Wylleman L 2008 A Petrov-type I and generically asymmetric rotating dust family Class. Quantum Grav. 25172001 